\title{
Review of Idealized Aircraft Wake Vortex Models
}

\author{
Nash'at N. Ahmad*, Fred H. Proctor ${ }^{\dagger}$ \\ NASA Langley Research Center, Hampton, Virginia, 23681 \\ Fanny M. Limon Duparcmeur \\ Craig Technologies Inc., Hampton, Virginia 23681 \\ Don Jacob ${ }^{\S}$ \\ Coherent Research Group LLC, Ormond Beach, Florida, 32174
}

Properties of three aircraft wake vortex models, Lamb-Oseen, Burnham-Hallock, and Proctor are reviewed. These idealized models are often used to initialize the aircraft wake vortex pair in large eddy simulations and in wake encounter hazard models, as well as to define matched filters for processing lidar observations of aircraft wake vortices. Basic parameters for each vortex model, such as peak tangential velocity and circulation strength as a function of vortex core radius size, are examined. The models are also compared using different vortex characterizations, such as the vorticity magnitude. Results of Euler and large eddy simulations are presented. The application of vortex models in the postprocessing of lidar observations is discussed.

\section{Nomenclature}

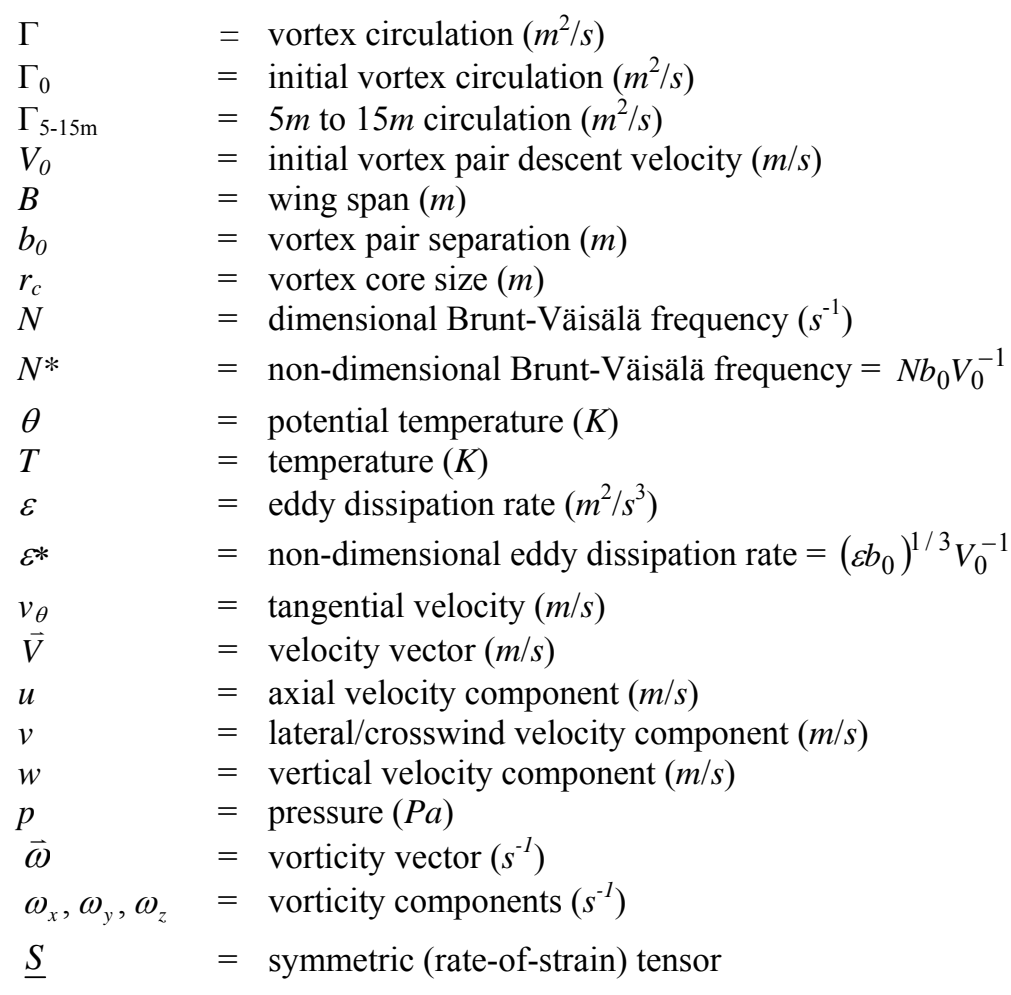

\footnotetext{
* Research Aerospace Engineer, NASA, Hampton, Virginia. Senior Member, AIAA.

${ }^{\dagger}$ Senior Scientist, NASA, Hampton, Virginia. Senior Member, AIAA.

${ }^{*}$ Research Scientist, Craig Technologies Inc., Hampton, Virginia. Member, AIAA.

${ }^{\S}$ Research Scientist, CRG, LLC, Ormond Beach, Florida. Member, AIAA.
} 


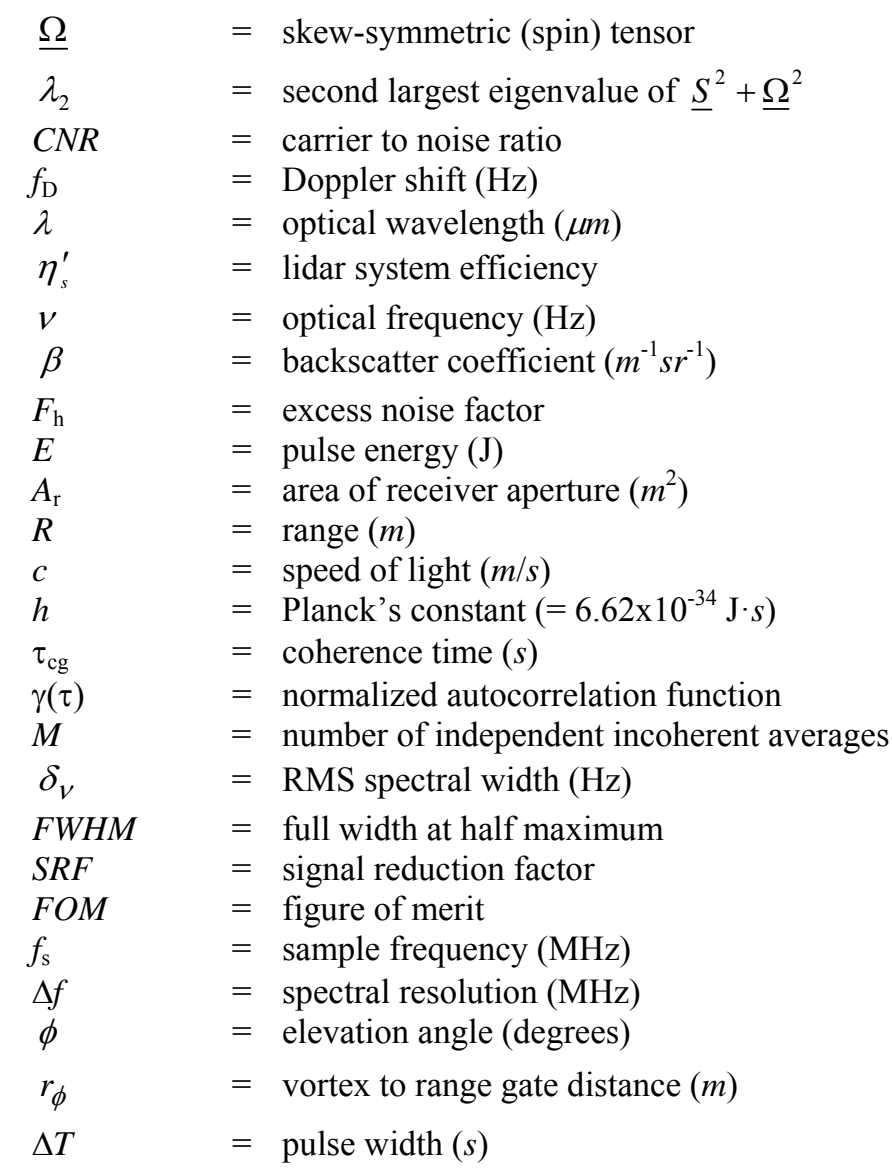

\section{Aircraft Vortex Models}

Three idealized vortex models are defined in this section. In the calculations presented below, the domain was defined by $(y, z) \in[-50,50] \times[0,100] \mathrm{m}$. The vortex was initialized at $\left(y_{0}, z_{0}\right)=(0,50) \mathrm{m}$. Unless stated otherwise, the aircraft parameters listed in Table 1 were used in this section. The parameters are based on elliptical wing loading.

Table 1: Aircraft Parameters

\begin{tabular}{|c|c|}
\hline Aircraft Parameter & B747-400 \\
\hline Wing Span, $B(m)$ & 64.43 \\
\hline Initial Vortex Pair Separation, $\boldsymbol{b}_{\mathbf{0}}=B \pi / 4(\mathrm{~m})$ & 50.60 \\
\hline Core Radius, $\boldsymbol{r}_{\mathrm{c}}(\mathrm{m})$ & $3.75 \sim 0.0582 B$ \\
\hline Initial Circulation, $\Gamma_{\mathbf{0}}\left(\mathrm{m}^{2} / \mathrm{s}\right)$ & 565 \\
\hline
\end{tabular}

\section{A. Lamb-Oseen Model}

The Lamb-Oseen vortex is an analytical solution of the Navier-Stokes equations (Appendix A). It has been used extensively for initializing large eddy simulations (Hennemann and Holzäpfel 2011; Misaka et al. 2012) and in the design of lidar matched filters. 
The tangential velocity, $v_{\theta}$ as a function of radial distance, $r$ from the vortex center in the Lamb-Oseen model is given by (Holzäpfel et al. 2000)

$$
v_{\theta}(r)=\frac{\Gamma_{0}}{2 \pi r}\left[1-\exp \left(-1.26\left(r / r_{C}\right)^{2}\right)\right]
$$

where $r_{\mathrm{c}}$ is the vortex core radius which is defined as the radius where the tangential velocity is maximum, $\Gamma_{0}$ is the vortex circulation, and $r$ is the distance from vortex center $\left(y_{0}, z_{0}\right)$

$$
r=\sqrt{\left(y-y_{0}\right)^{2}+\left(z-z_{0}\right)^{2}}
$$

The tangential velocity in Eq. (1) can be converted to Cartesian velocities using the following relations

$$
\begin{aligned}
& v(y, z)=(\operatorname{sign}) v_{\theta} \frac{z}{r} \\
& w(y, z)=-(\operatorname{sign}) v_{\theta} \frac{y}{r}
\end{aligned}
$$

where sign is used to define the direction of vortex rotation, +1 for clockwise and -1 for counter-clockwise direction.

The Cartesian components in Eq. (3) can be combined and re-written in terms of $v_{\theta}$

$$
v_{\theta}=\sqrt{v^{2}+w^{2}}
$$

Figure 1 shows the tangential velocity field for the Lamb-Oseen model. In this example, a B747 was used, and the vortex core radius size was set to $3.75 \mathrm{~m}$.

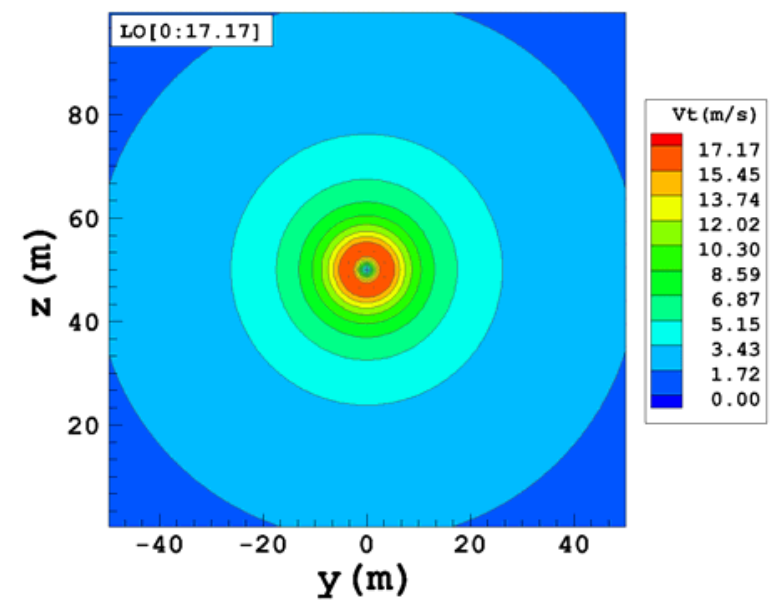

Figure 1. Lamb-Oseen (LO) Vortex. Tangential Velocity $(\mathrm{m} / \mathrm{s}) . r_{\mathrm{c}}=3.75 \mathrm{~m}$. The resolution of the underlying grid is: $\Delta y=\Delta z=0.5 \mathrm{~m}$. B747-400 $\Gamma_{0}=565 \mathrm{~m}^{2} / \mathrm{s}$.

\section{B. Burnham-Hallock Model}

The Burnham-Hallock model (Burnham and Hallock 1982) is given by Eq. (5). It is the most widely used model for wake vortex applications, which include processing of lidar observations, initialization of large eddy simulations (De Visscher et al. 2013), and modeling of aircraft response to wake encounters (Reimer and Vicroy 1996; Schwarz et al. 2010). Figure 2 shows the tangential velocity field given by

$$
v_{\theta}(r)=\frac{\Gamma_{0}}{2 \pi r} \frac{r^{2}}{r^{2}+r_{c}^{2}} .
$$




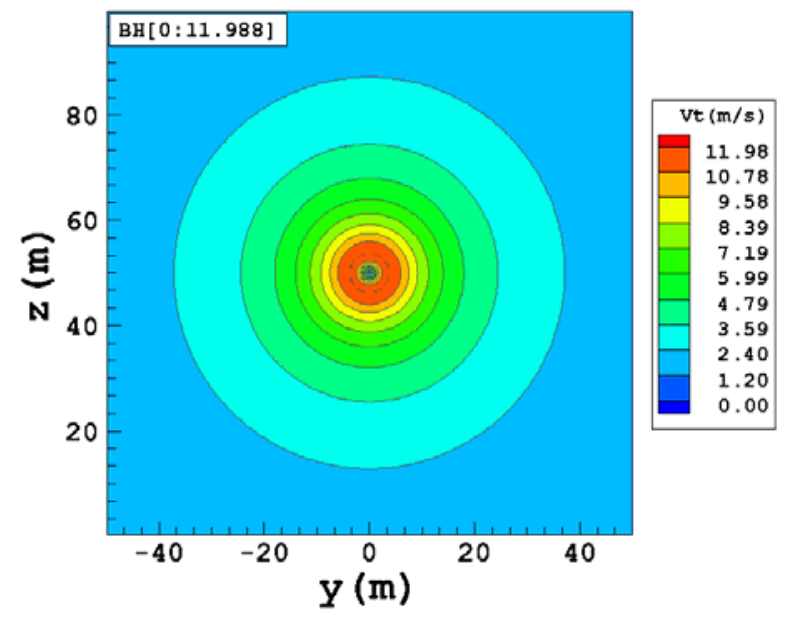

Figure 2. Burnham-Hallock (BH) Vortex. Tangential Velocity $(\mathrm{m} / \mathrm{s}) . \quad r_{\mathrm{c}}=3.75 \mathrm{~m}$. The resolution of the underlying grid is: $\Delta y=\Delta z=0.5 \mathrm{~m}$. B747-400 $\Gamma_{0}=565 \mathrm{~m}^{2} / \mathrm{s}$.

\section{Proctor Model}

The Proctor vortex model (Proctor 2000) is based on a detailed analysis of lidar observations of vortex tangential velocity, and is given by Eq. (6). This model has been used for the initialization of large eddy simulations of wake vortex decay in stratified and turbulent environments. Figure 3 shows the tangential velocity field of the Proctor model given by

$$
v_{\theta}(r)=\left\{\begin{array}{cc}
1.0939 \frac{\Gamma_{0}}{2 \pi r}\left[1-\exp \left(-10\left(1.4 r_{C} / B\right)^{0.75}\right)\right] \times\left[1-\exp \left(-1.2527\left(r / r_{C}\right)^{2}\right)\right] & \text { if } r \leq 1.4 r_{C} \\
\frac{\Gamma_{0}}{2 \pi r}\left[1-\exp \left(-10(r / B)^{0.75}\right)\right] & \text { if } r>1.4 r_{C}
\end{array} .\right.
$$

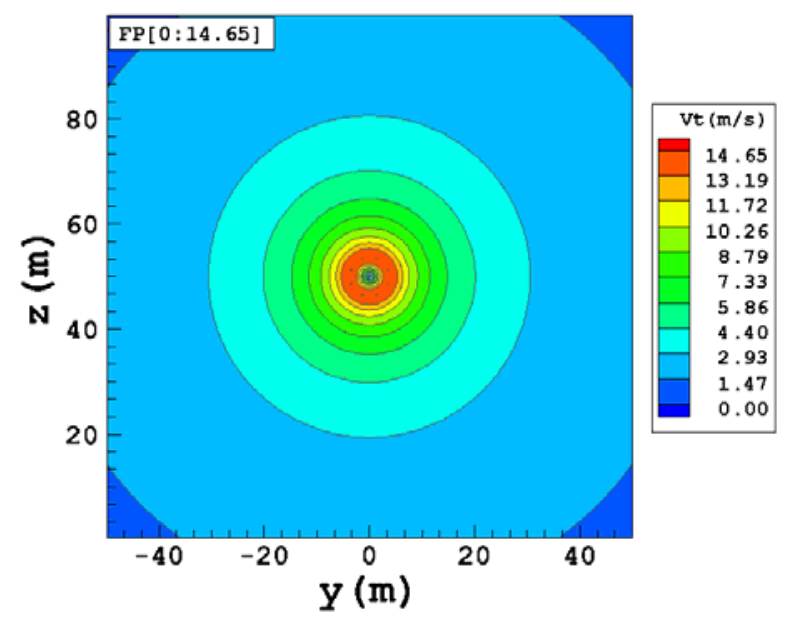

Figure 3. Proctor (FP) Vortex. Tangential Velocity $(\mathrm{m} / \mathrm{s}) . \quad r_{\mathrm{c}}=3.75 \mathrm{~m}$. The resolution of the underlying grid is: $\Delta y=\Delta z=0.5 \mathrm{~m}$. B747-400 $\Gamma_{0}=565 \mathrm{~m}^{2} / \mathrm{s}$.

The three vortex models described in this paper are not the only vortex models that have been used for wake applications; however, they are the most widely used. Other examples include the Winckelmans (Winckelmans et al. 2000), the Jacquin, and the Rankine vortex models (Gerz et al. 2002). 


\section{Comparison of Vortex Models}

Basic characteristics of vortex models are compared in this section: 1) magnitude and distribution of tangential and vertical velocity components, 2) circulation strength, and 3) tangential velocity magnitude and distribution as a function of the vortex core radius size.

\section{A. Velocity Components}

Comparisons of the tangential and vertical velocity components of the three models along the vortex centerline are shown in Figure 4 and Figure 5, respectively. The crosswind velocity, $v$ along the vortex centerline $(z=50 \mathrm{~m})$ is zero.

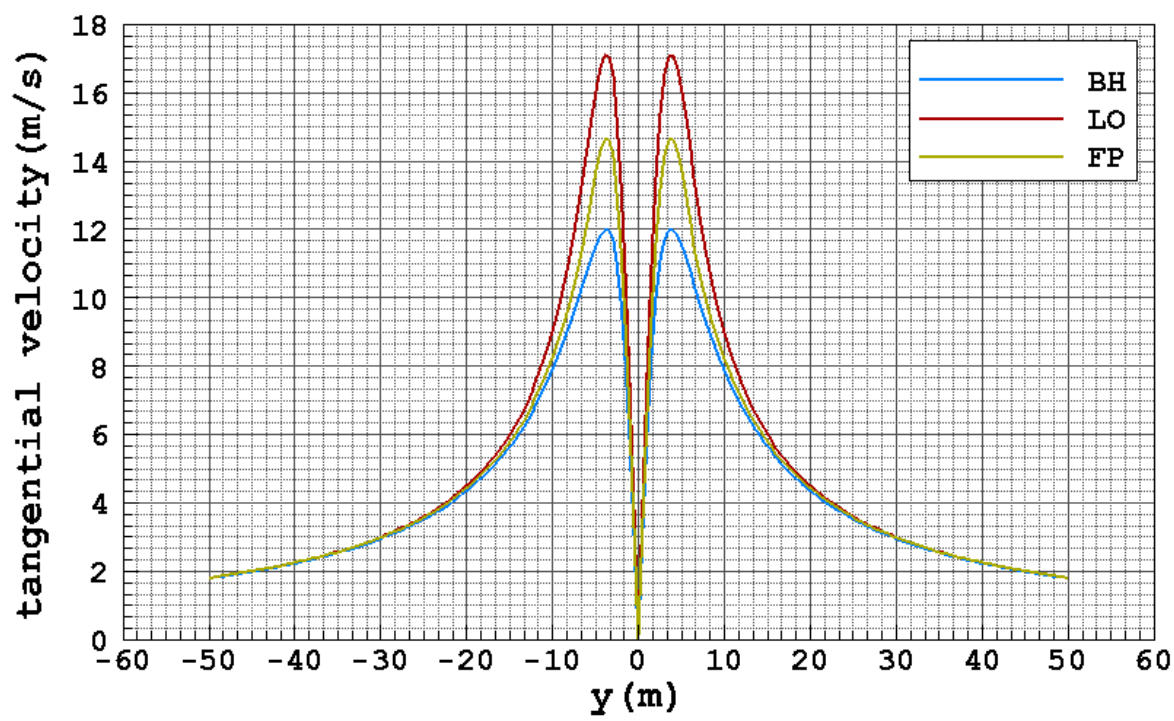

Figure 4. Comparison of the tangential velocity component $(\mathrm{m} / \mathrm{s})$ along the vortex centerline $(\mathrm{z}=50 \mathrm{~m})$, and is the same profile that would be observed through any line through the vortex center - circularly symmetric. $r_{\mathrm{c}}=3.75 \mathrm{~m} . \mathrm{B} 747-400 \Longrightarrow \Gamma_{0}=565 \mathrm{~m}^{2} / \mathrm{s}$.

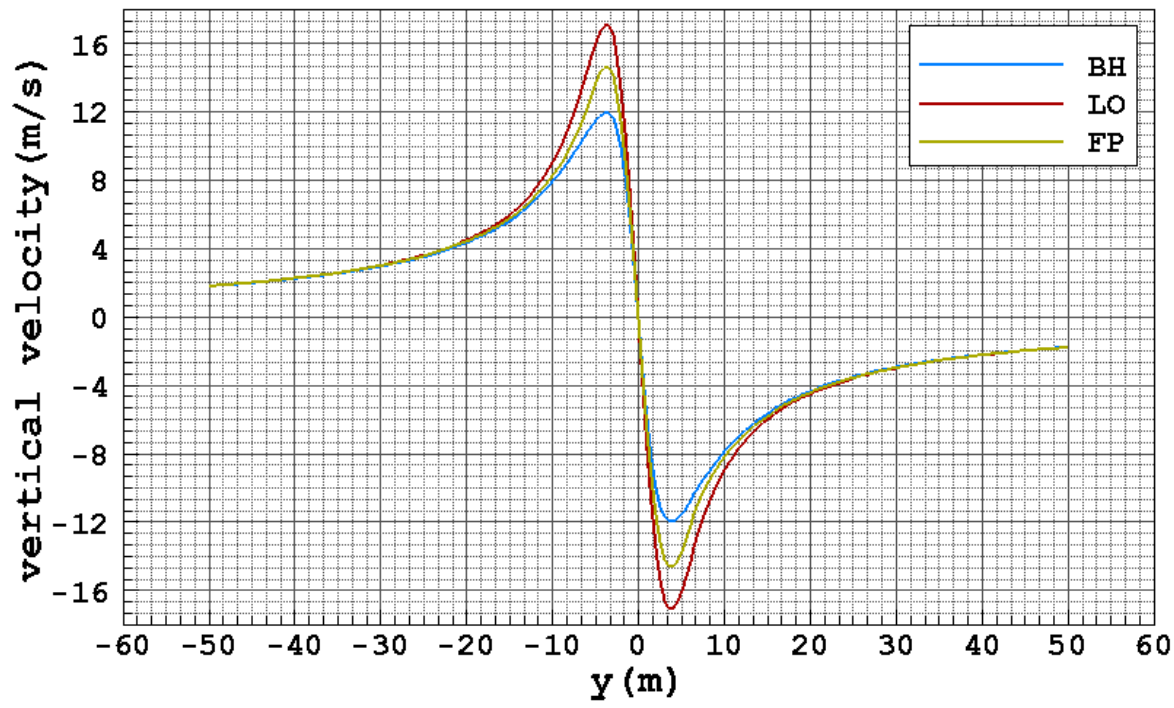

Figure 5. Comparison of the vertical velocity component $(\mathrm{m} / \mathrm{s})$ along the vortex centerline for $\boldsymbol{r}_{\mathrm{c}}=3.75 \mathrm{~m}$. B747-400 $\Gamma_{0}=565 \mathrm{~m}^{2} / \mathrm{s}$. 


\section{B. Circulation}

The circulation of a single vortex as a function of radial distance from the vortex center can be defined in terms of the tangential velocity

$$
\Gamma(r)=2 \pi r v_{\theta}(r) .
$$

Eq. (7) for the three vortex models is plotted in Figure 6 using vortex core radius values of $3.75 \mathrm{~m}$ and $1.28 \mathrm{~m}$. $v_{\theta}(r)$ in Eq. (7) was calculated using Eq. (1), (5) and (6) for the three vortex models. The region between 5-15m has been marked in the figure for reference. Average circulation between $5-15 \mathrm{~m}$ is often used to characterize wake hazard (Burnham and Hallock 2013). Hazard circulation is discussed in Section V.
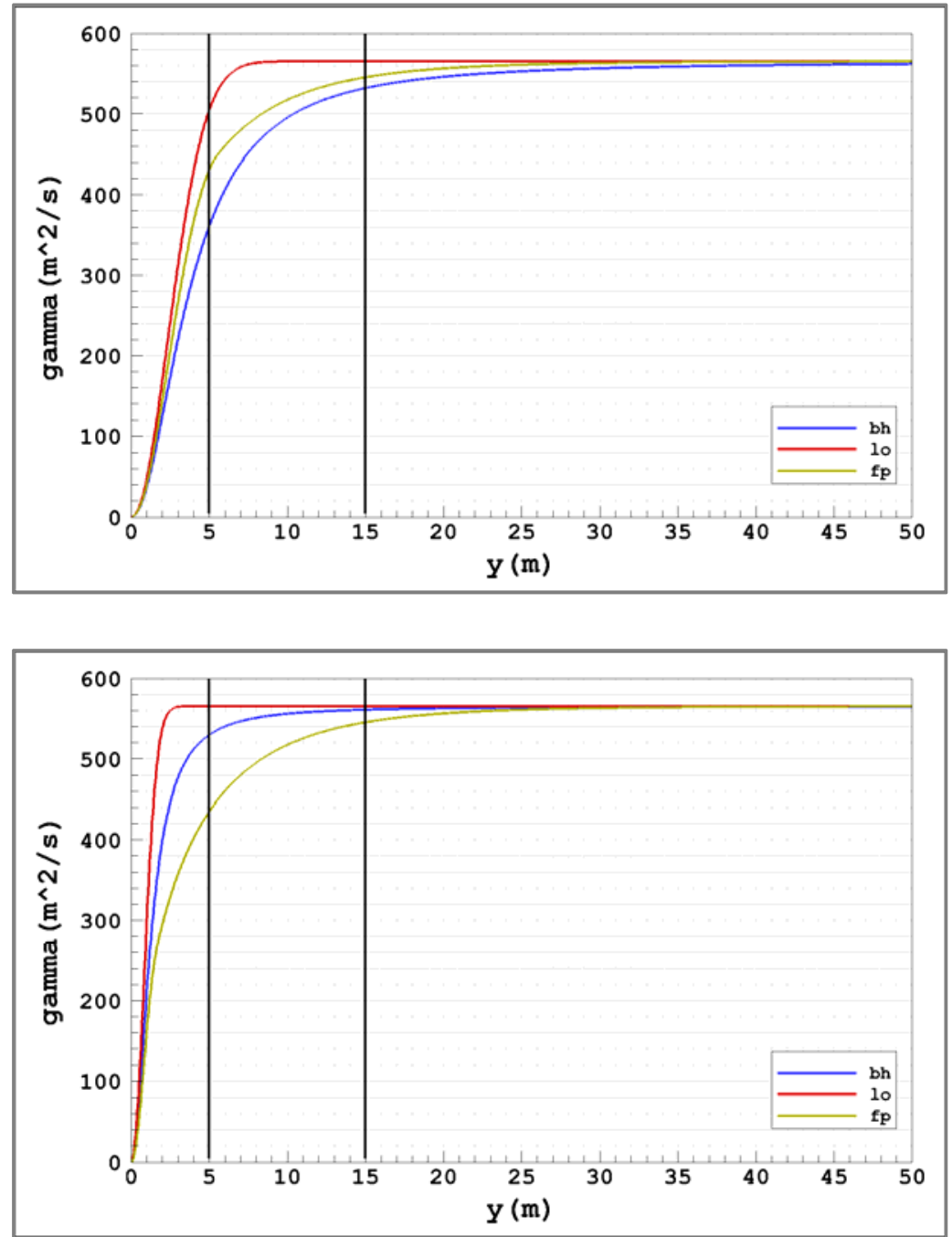

Figure 6. Comparison of circulation $\left(\mathrm{m}^{2} / \mathrm{s}\right)$ along the vortex centerline. $r_{\mathrm{c}}=3.75 \mathrm{~m}$ (top) and $r_{\mathrm{c}}=1.28 \mathrm{~m}$ (bottom). B747-400 $\Gamma_{0}=565 \mathrm{~m}^{2} / \mathrm{s}$.

\section{Vortex Core Radius}

A wide range of values for the vortex core radius have been suggested in the literature. These values range anywhere from $1 \%$ of the wing span (Delisi et al. 2003) to 5\% of the wing span (Schwarz et al. 2010). Large eddy simulations can use values in the range of $7 \%$ of the wing span due to computational constraints. The tangential velocities for different models and for different values of core radius ranging from $1 \%$ to $6.9 \%$ of the wing span are shown in Figure 7. 

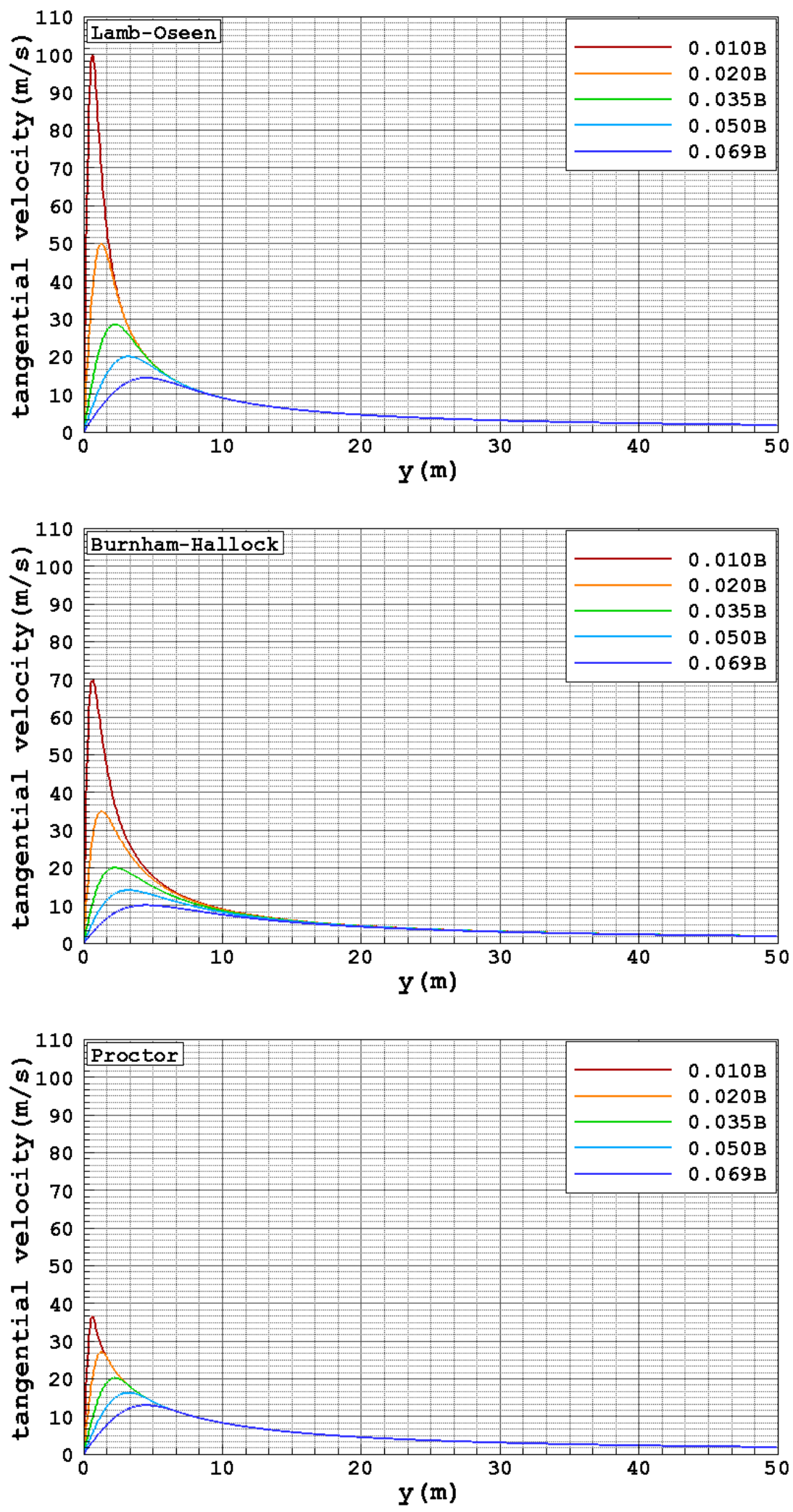

Figure 7. Tangential velocity $(\mathrm{m} / \mathrm{s})$ calculation for different core sizes for the three vortex models. Aircraft is a B747-400 $\Gamma_{0}=565 \mathrm{~m}^{2} / \mathrm{s} . \quad B=64.43 \mathrm{~m}$. 


\section{Vortex Characterizations} by

Several methods have been used for vortex characterization (Jiang et al. 2004). The vorticity vector, $\vec{\omega}$ is given

$$
\vec{\omega}=\nabla \times \vec{V}=\operatorname{det}\left|\begin{array}{ccc}
\hat{e}_{x} & \hat{e}_{y} & \hat{e}_{z} \\
\frac{\partial}{\partial x} & \frac{\partial}{\partial y} & \frac{\partial}{\partial z} \\
u & v & w
\end{array}\right|=\left[\begin{array}{l}
\partial w / \partial y-\partial v / \partial z \\
\partial u / \partial z-\partial w / \partial x \\
\partial v / \partial x-\partial u / \partial y
\end{array}\right]=\left[\begin{array}{c}
\omega_{x} \\
\omega_{y} \\
\omega_{z}
\end{array}\right],
$$

where $\omega_{x}, \omega_{y}$, and $\omega_{z}$ are the three components of vorticity in $x, y$ and $z$-direction. In two dimensions, the vorticity has only one component (Figure 8 - left panel). The vorticity, $\nabla \times \vec{V}$ is zero around the vortex except near the vortex center where a spike in vorticity is observed. The divergence property $(\nabla \cdot \vec{V}=0)$ is satisfied by all three models. Jeong and Hussain (1995) define the extent of a vortex based on the second largest eigenvalue, $\lambda_{2}$ of $\underline{S}^{2}+\underline{\Omega}^{2}$, where $\underline{S}$ is the rate-of-strain tensor and $\underline{\Omega}$ is the spin tensor (Appendix B). A vortex is defined as the region in which $\lambda_{2}$ is negative. Figure 8 (right panel) shows the comparison of $\lambda_{2}$ for the three vortex models along the centerline. All three models have similar vortex region definition in $\lambda_{2}$.
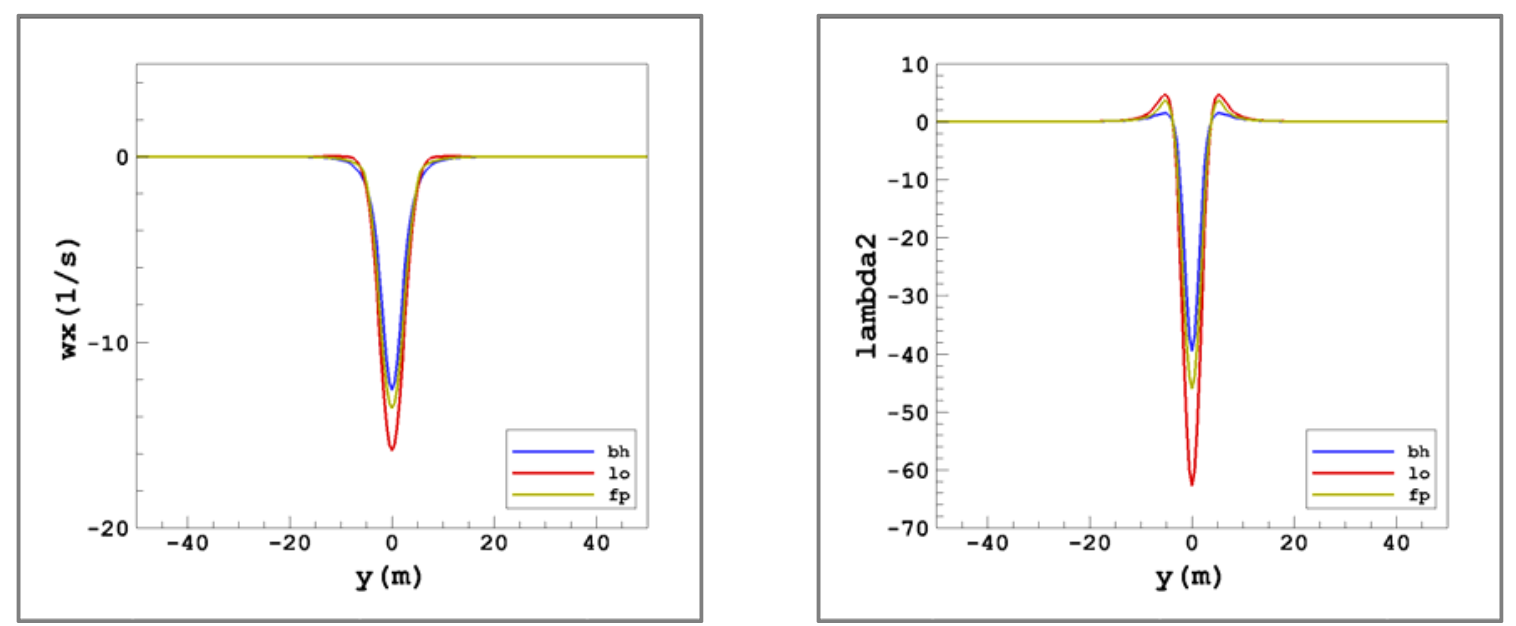

Figure 8. Comparison of vorticity, $\omega_{\mathrm{x}}\left(s^{-1}\right)$ along the vortex centerline (left panel) and the second largest eigenvalue of $\underline{S}^{2}+\underline{\Omega}^{2}, \lambda_{2}$ (right panel) for $r_{\mathrm{c}}=3.75 \mathrm{~m}$. B747-400. $\Gamma_{0}=565 \mathrm{~m}^{2} / \mathrm{s}$.

\section{Euler Solutions of Vortex Pair Descent in Stratified Atmosphere}

The descent of an idealized vortex pair in a non-turbulent stratified atmosphere was simulated using a twodimensional Euler code based on a high-resolution wave propagation method (Ahmad and Lindeman 1997). The governing equations for atmospheric flows in two dimensions are given by:

$$
\frac{\partial U}{\partial t}+\frac{\partial G}{\partial y}+\frac{\partial H}{\partial z}=\frac{\partial G_{v}}{\partial y}+\frac{\partial H_{v}}{\partial z}+\psi+S
$$

where

$$
U=\left[\begin{array}{c}
\rho \\
\rho v \\
\rho w \\
\rho \theta
\end{array}\right], \quad G=\left[\begin{array}{c}
\rho v \\
\rho v^{2}+p \\
\rho v w \\
v \rho \theta
\end{array}\right], \quad H=\left[\begin{array}{c}
\rho w \\
\rho v w \\
\rho w^{2}+p \\
w \rho \theta
\end{array}\right], \quad \psi=\left[\begin{array}{c}
0 \\
0 \\
\rho g \\
0
\end{array}\right] .
$$


In Eq. (9)-(10), $\rho$ is the density of air, $v$ is the velocity component in the $y$-direction (crosswind direction), $w$ is the velocity component in the $z$-direction, $p$ is the pressure, $\theta$ is the potential temperature, and $g$ is the acceleration due to gravity. The system is closed by an equation of state for pressure,

$$
p=C_{0}(\rho \theta)^{\gamma}
$$

where $C_{0}$ is a constant given by:

$$
C_{0}=\frac{R_{d}^{\gamma}}{p_{0}^{R_{d} / C_{v}}} .
$$

In the above relations, $\gamma$ is the ratio of specific heats, and $R_{d}$ is the gas constant for dry air. $C_{p}$ and $C_{v}$ are the specific heats of air at constant pressure and volume respectively. $p_{0}$ is the reference base-state pressure. In these simulations, the atmosphere was assumed to be dry, and the source term $S$ was set to zero ( $S$ represents heat sinks and sources due to microphysical processes of cloud formation/dissipation and atmospheric radiation transfer). The viscous fluxes, $G_{\mathrm{v}}$ and $H_{\mathrm{v}}$ were also set to zero, and only the Euler solutions were considered. A high-resolution wave propagation method based on flux-wave decomposition (LeVeque 2002; Ahmad and Lindeman 2007) was used to compute Eq. (9)-(12).

The computational domain was defined by $(y, z) \in[-300,300] \times[0,600] m$ with $t \in[0,60] \mathrm{s}$. The mesh had a resolution of $\Delta y=\Delta z=0.3 \mathrm{~m}$ ( $2000 \times 2000$ cells). The wake vortex pair was approximated by the superposition of velocity fields of two counter-rotating vortices defined by the idealized vortex models. The vortex pair was initialized at $\left(y_{0}, z_{0}\right)=(0,300) \mathrm{m}$. The vortex core radius, $r_{\mathrm{c}}$ was set to $2.255 \mathrm{~m}(\sim 3.5 \%$ of the wing span), and the initial circulation strength, $\Gamma_{0}$ was set to $565 \mathrm{~m}^{2} / \mathrm{s}$ (B747-400). The background atmospheric stability was set to zero $\left(N^{*}=0\right)$. Open/farfield boundary conditions were used in the lateral and at the top. The bottom boundary was set to solid wall. The simulations were run for a final time of $60 \mathrm{~s}$.

Figure 9 shows the computed velocity and pressure fields at time $=60 \mathrm{~s}$. The domain maximum and minimum velocities and pressure values are compared in Table 2. The Lamb-Oseen model has the highest pressure deficit. Also, the maximum and minimum velocity values predicted by the Lamb-Oseen initialization have larger magnitudes compared to the Burnham-Hallock and Proctor models. The differences in simulation results using the Proctor and the Burnham-Hallock models for initialization were relatively small. The initial descent velocity, $V_{0}$ of the vortex pair in all three simulations was $1.78 \mathrm{~m} / \mathrm{s}$ compared to $1.777 \mathrm{~m} / \mathrm{s}$ estimated from measurements.

Table 2: Vortex Pair Descent: Comparison of the models after $60 \mathrm{~s}-\boldsymbol{r}_{\mathrm{c}}=\mathbf{2 . 2 5 5 \mathrm { m }}$

\begin{tabular}{|c|c|c|c|c|c|c|}
\hline model & $\boldsymbol{v}_{\min }(\boldsymbol{m} / \mathbf{s})$ & $\boldsymbol{v}_{\max }(\boldsymbol{m} / \mathbf{s})$ & $\boldsymbol{w}_{\min }(\mathbf{m} / \mathbf{s})$ & $\boldsymbol{w}_{\max }(\mathbf{m} / \mathbf{s})$ & $\boldsymbol{p}_{\min }(\mathbf{P a})$ & $\boldsymbol{p}_{\max }(\mathbf{P a})$ \\
\hline $\mathbf{L O}$ & -13.66 & 13.66 & -15.96 & 12.32 & -340.73 & 12.03 \\
\hline $\mathbf{B H}$ & -12.01 & 12.01 & -14.32 & 10.72 & -270.30 & 11.97 \\
\hline $\mathbf{F P}$ & -11.45 & 11.45 & -13.76 & 10.17 & -248.59 & 11.98 \\
\hline
\end{tabular}



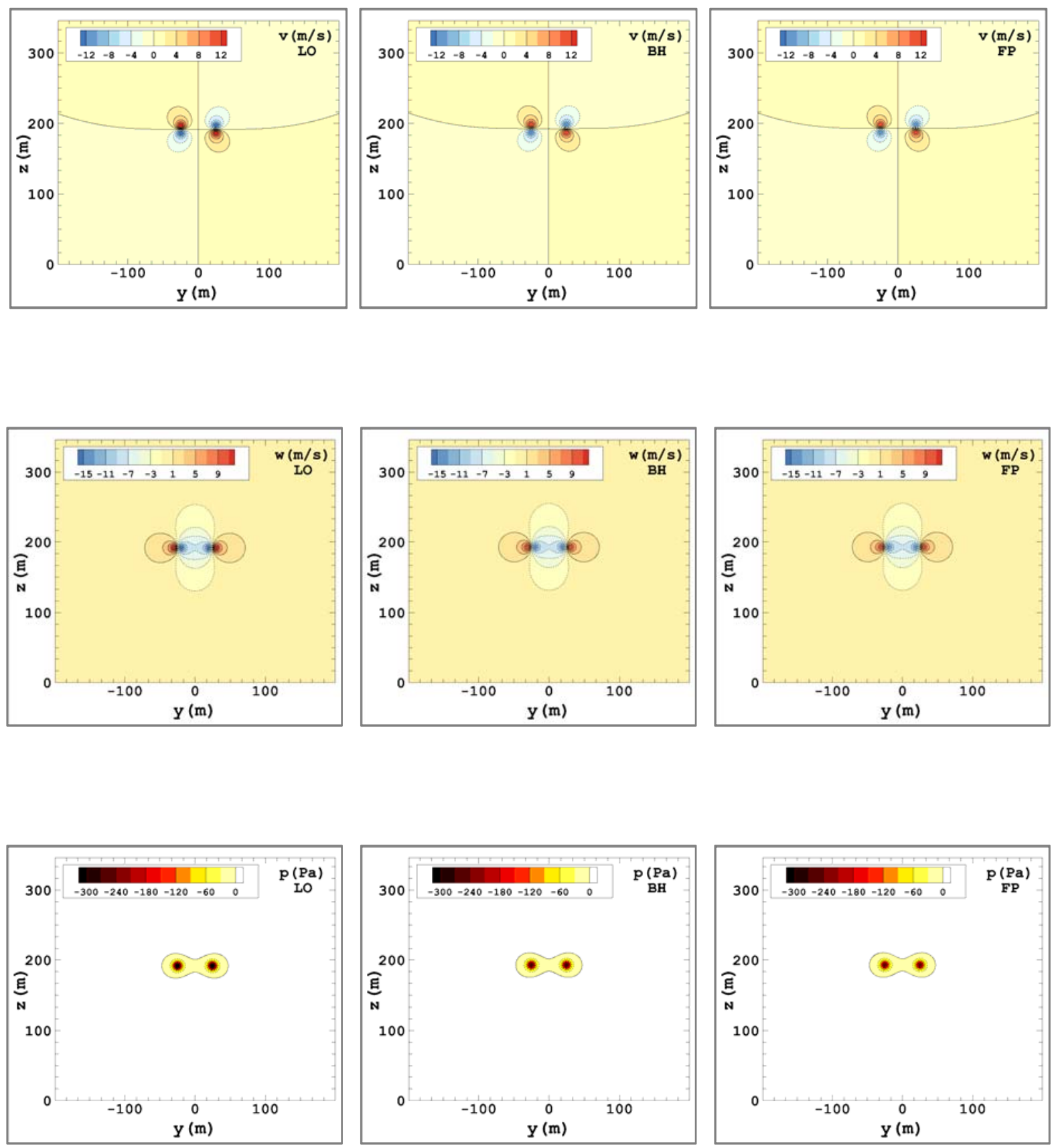

Figure 9. Vortex pair velocity and pressure fields at time $=60 \mathrm{~s}$. $v$-velocity $(\mathrm{m} / \mathrm{s})$ is shown in the top panel, $w$ velocity $(\mathrm{m} / \mathrm{s})$ is shown in the middle panel, and pressure $(\mathrm{Pa})$ is shown in the bottom panel. Aircraft is a B747-400 $\Gamma_{0}=565 \mathrm{~m}^{2} / \mathrm{s}$. $B=64.43 \mathrm{~m}$. The vortex core radius, $r_{\mathrm{c}}=2.255 \mathrm{~m}(\sim 3.5 \%$ of $B)$. 


\section{Vortex Pair Descent in Stratified Turbulent Atmosphere}

In this section, the three idealized vortex models were used to initialize large eddy simulations using the Terminal Area Simulation System (TASS). TASS (Proctor 1987) is a cloud-resolving model which has been used successfully for simulating a wide range of atmospheric flow applications - microburst and wind shear (Proctor and Bowles 1992); transport and decay of aircraft wakes in turbulent atmosphere (Proctor 1996; Han et al. 2000a; Han et al. 2000b); sensor characterization (Lai et al. 2010), and convection induced turbulence (Proctor et al. 2002; Ahmad and Proctor 2011). TASS computes the primitive variables non-hydrostatic equations in three dimensions and is capable of resolving flows at multiple spatial (grid resolutions varying from less than $1 \mathrm{~m}$ to $2 \mathrm{~km}$ in the horizontal) and temporal scales (few seconds in the case of turbulence eddies to hours for long-lived convective phenomena). The model solves prognostic equations for potential temperature, water vapor, cloud droplets, ice crystals, rain, snow, and hail and includes a microphysics package for cloud and precipitation development. Subgrid scale diffusion is parameterized via a Smagorinsky-type turbulence closure (Smagorinsky 1963), and surface layer processes are computed based on the Monin-Obukhov similarity theory. The Smagorinsky model implemented in TASS includes a rotation correction for vortical flows (Han et al. 2000b) when simulating the decay and transport aircraft wakes.

The TASS model equations are discretized using fourth-order finite-differences in space for the calculation of momentum and pressure fields, and the third-order Leonard scheme (Leonard 1995) is used to calculate the transport of potential temperature and water vapor. The Klemp-Wilhelmson time-splitting scheme (Klemp and Wilhelmson 1978 ) is used for computational efficiency in which the higher-frequency terms given in the left hand side of Eq. (13) and (20) are integrated by enforcing the CFL criteria to take into account sound wave propagation due to compressibility effects. The remaining terms in Eq. (14), Eq. (20) and Eq. (21) are integrated using a larger time step that is appropriate for anelastic and incompressible flows. Non-reflecting Orlanski boundary conditions (Orlanski 1976) are imposed at the outflow boundaries. A sixth-order filter is used to damp-out spurious oscillations that may arise due to the use of centered-differencing of momentum and pressure terms. The TASS model equations with the assumption of a dry atmosphere can be written as follows

$$
\frac{\partial u_{i}}{\partial t}+\frac{H}{\rho_{0}} \frac{\partial p^{\prime}}{\partial x_{i}}=-\frac{\partial u_{i} u_{j}}{\partial x_{j}}+u_{i} \frac{\partial u_{j}}{\partial x_{j}}+g(H-1) \delta_{i 3}+\frac{1}{\rho_{0}} \frac{\partial \tau_{i j}}{\partial x_{j}},
$$

where $u_{\mathrm{i}}$ and $u_{\mathrm{j}}$ are the velocity components, $x_{\mathrm{i}}$ and $x_{\mathrm{j}}$ are the spatial dimensions, $p$ is the pressure, $g$ is the acceleration due to gravity, $\tau_{\mathrm{ij}}$ is the shear stress tensor, and $\delta$ is the Kronecker delta. $H$ is the density ratio defined by

$$
H \equiv \frac{\rho_{0}}{\rho}=\frac{\theta}{\theta_{0}}\left(\frac{p_{0}}{p}\right)^{C_{V} / C_{p}},
$$

In Eq. (14), $C_{p}\left(=1004 \mathrm{~J} \mathrm{~K}^{-1} \mathrm{~kg}^{-1}\right)$ and $C_{v}\left(=717 \mathrm{~J} \mathrm{~K}^{-1} \mathrm{~kg}^{-1}\right)$ are the specific heats of air at constant pressure and volume, respectively. $p_{0}$ is the base-state pressure, $\rho_{0}$ is the base-state density, and $\theta_{0}$ is the base-state potential temperature. The horizontal velocities, atmospheric pressure, potential temperature, and density are decomposed into initial time-invariant hydrostatic base-states $\left(u_{0}, v_{0}, p_{0}, \theta_{0}, \rho_{0}\right)$ which are a function of height only and the time-variant perturbations $\left(u^{\prime}, v^{\prime}, p^{\prime}, \theta^{\prime}, \rho^{\prime}\right)$ which are a function of both space and time:

$$
\begin{gathered}
u(x, y, z, t)=u_{0}(z)+u^{\prime}(x, y, z, t) \\
v(x, y, z, t)=v_{0}(z)+v^{\prime}(x, y, z, t) \\
p(x, y, z, t)=p_{0}(z)+p^{\prime}(x, y, z, t) \\
\theta(x, y, z, t)=\theta_{0}(z)+\theta^{\prime}(x, y, z, t) \\
\rho(x, y, z, t)=\rho_{0}(z)+\rho^{\prime}(x, y, z, t),
\end{gathered}
$$

The prognostic equation for pressure perturbation is approximated by 


$$
\frac{\partial p^{\prime}}{\partial t}+\frac{C_{p}}{C_{v}} p \frac{\partial u_{j}}{\partial x_{j}}=\rho_{0} g u_{j} \delta_{j 3} .
$$

The conservation of energy is given in terms of potential temperature with the assumption of a dry adiabatic atmosphere

$$
\frac{\partial \theta}{\partial t}=-\frac{\partial u_{j} \theta}{\partial x_{j}}+\theta \frac{\partial u_{j}}{\partial x_{j}} .
$$

The system is closed by an equation of state for pressure

$$
p=R_{d} \rho T .
$$

Simulations were run for two different aircraft (B747 and B767). The first step in TASS wake simulations is the generation of background homogeneous turbulence. The methodology is described in Han et al. (2000b). A low background turbulence field was generated $\left(\varepsilon^{*}=0.07\right)$ for both simulation sets, and the atmospheric stability was set to neutral $\left(N^{*}=0\right)$. The mesh had a resolution of $\Delta x=2 \mathrm{~m}$, and $\Delta y=\Delta z=1.5 \mathrm{~m}$ for both cases. The vortex core radius, $r_{\mathrm{c}}$ was set to $4.5 \mathrm{~m}$ in B747 and $3 \mathrm{~m}$ in B767 simulations. The choice of $r_{\mathrm{c}}$ depends on mesh resolution, and the computational requirements for lower values of $r_{\mathrm{c}}$ can be restrictive. Periodic boundary conditions were used in all directions. For each aircraft, three simulations were run, each initialized with different vortex model.

Figures (10)-(11) show the $\lambda_{2}$ isosurfaces for the two simulation sets at different times. The theoretical value of vortex pair time to link, $T_{\mathrm{L}}$ based on Crow and Bates (1976) and modified by Sarpkaya et al. (2001) is given by

$$
\begin{array}{ll}
T_{L}=9 & \text { if } \varepsilon^{*}<0.001 \\
T_{L}=9.18-180 \varepsilon^{*} & \text { if } \quad 0.001 \leq \varepsilon^{*}<0.0121 \\
T_{L}=-1.5583 \ln \left(\varepsilon^{*}\right)+0.1556 & \text { if } \quad 0.0121 \leq \varepsilon^{*}<0.2535 \\
T_{L}=\left(\frac{0.7474}{\varepsilon^{*}}\right)^{3 / 4} & \text { if } \quad \varepsilon^{*} \geq 0.2535
\end{array}
$$

The time to link for the two cases is compared with the theoretical value in Tables 3-4. Overall the values compare well with the theoretical value for all three vortex initializations. The Lamb-Oseen initialization has the fastest $T_{\mathrm{L}}$, and the simulations initialized with the Burnham-Hallock model predict values that are closest to the theoretical value. A comparison of domain maximum and minimum values of velocity and pressure fields at $60 \mathrm{~s}$ and 120 s is given in Tables 5-8.

Table 3: Time to Link, $T_{\mathrm{L}}$ (B747)

\begin{tabular}{|c|c|}
\hline Initialization & $\boldsymbol{T}_{\mathbf{L}}$ \\
\hline Lamb-Oseen (LO) & 3.92 \\
\hline Burnham-Hallock (BH) & 4.17 \\
\hline Proctor (FP) & 4.01 \\
\hline Theoretical (Eq. 23) & 4.29 \\
\hline
\end{tabular}

Table 4: Time to Link, $T_{\mathrm{L}}$ (B767)

\begin{tabular}{|c|c|}
\hline Initialization & $\boldsymbol{T}_{\mathbf{L}}$ \\
\hline Lamb-Oseen (LO) & 3.82 \\
\hline Burnham-Hallock (BH) & 4.13 \\
\hline Proctor (FP) & 3.92 \\
\hline Theoretical (Eq. 23) & 4.29 \\
\hline
\end{tabular}


Table 5: Vortex Decay (B747): Comparison of the models after 60s $-r_{\mathrm{c}}=4.5 \mathrm{~m}$

\begin{tabular}{|c|c|c|c|c|c|c|}
\hline model & $\boldsymbol{v}_{\min }(\boldsymbol{m} / \mathbf{s})$ & $\boldsymbol{v}_{\max }(\boldsymbol{m} / \mathbf{s})$ & $\boldsymbol{w}_{\min }(\boldsymbol{m} / \mathbf{s})$ & $\boldsymbol{w}_{\max }(\boldsymbol{m} / \mathbf{s})$ & $\boldsymbol{p}_{\min }(\mathbf{P a})$ & $\boldsymbol{p}_{\max }(\mathbf{P a})$ \\
\hline $\mathbf{L O}$ & -9.363 & 9.342 & -11.832 & 7.938 & -177.453 & 2.691 \\
\hline $\mathbf{B H}$ & -8.066 & 8.359 & -10.334 & 6.846 & -133.842 & 1.912 \\
\hline $\mathbf{F P}$ & -8.983 & 9.037 & -11.246 & 7.511 & -161.874 & 2.301 \\
\hline
\end{tabular}

Table 6: Vortex Decay (B747): Comparison of the models after 120s $-r_{c}=4.5 m$

\begin{tabular}{|c|c|c|c|c|c|c|}
\hline model & $\boldsymbol{v}_{\min }(\boldsymbol{m} / \mathbf{s})$ & $\boldsymbol{v}_{\max }(\boldsymbol{m} / \mathbf{s})$ & $\boldsymbol{w}_{\min }(\mathbf{m} / \mathbf{s})$ & $\boldsymbol{w}_{\max }(\mathbf{m} / \mathbf{s})$ & $\boldsymbol{p}_{\min }(\mathbf{P a})$ & $\boldsymbol{p}_{\max }(\mathbf{P a})$ \\
\hline $\mathbf{L O}$ & -9.356 & 10.424 & -13.866 & 8.578 & -152.349 & 8.251 \\
\hline $\mathbf{B H}$ & -7.845 & 8.292 & -12.775 & 6.726 & -115.079 & 6.700 \\
\hline FP & -9.934 & 9.084 & -13.709 & 7.442 & -140.507 & 5.826 \\
\hline
\end{tabular}

Table 7: Vortex Decay (B767): Comparison of the models after 60s $-r_{c}=3 m$

\begin{tabular}{|c|c|c|c|c|c|c|}
\hline model & $\boldsymbol{v}_{\min }(\boldsymbol{m} / \mathbf{s})$ & $\boldsymbol{v}_{\max }(\boldsymbol{m} / \mathbf{s})$ & $\boldsymbol{w}_{\min }(\mathbf{m} / \mathbf{s})$ & $\boldsymbol{w}_{\max }(\boldsymbol{m} / \mathbf{s})$ & $\boldsymbol{p}_{\min }(\mathbf{P a})$ & $\boldsymbol{p}_{\max }(\mathbf{P a})$ \\
\hline $\mathbf{L O}$ & -6.651 & 6.759 & -8.994 & 5.561 & -87.910 & 3.049 \\
\hline $\mathbf{B H}$ & -6.221 & 6.391 & -8.505 & 5.183 & -77.393 & 2.747 \\
\hline FP & -6.547 & 6.690 & -8.978 & 5.409 & -84.917 & 2.864 \\
\hline
\end{tabular}

Table 8: Vortex Decay (B767): Comparison of the models after 120s $-r_{\mathrm{c}}=3 m$

\begin{tabular}{|c|c|c|c|c|c|c|}
\hline model & $\boldsymbol{v}_{\min }(\boldsymbol{m} / \mathbf{s})$ & $\boldsymbol{v}_{\max }(\boldsymbol{m} / \mathbf{s})$ & $\boldsymbol{w}_{\min }(\mathbf{m} / \mathbf{s})$ & $\boldsymbol{w}_{\max }(\mathbf{m} / \mathbf{s})$ & $\boldsymbol{p}_{\min }(\mathbf{P a})$ & $\boldsymbol{p}_{\max }(\mathbf{P a})$ \\
\hline $\mathbf{L O}$ & -7.242 & 8.416 & -7.890 & 6.341 & -70.409 & 2.128 \\
\hline $\mathbf{B H}$ & -5.793 & 6.301 & -7.655 & 4.731 & -64.298 & 1.559 \\
\hline FP & -6.647 & 6.693 & -7.322 & 4.556 & -69.904 & 1.904 \\
\hline
\end{tabular}


Lamb-Oseen Initialization (120s)

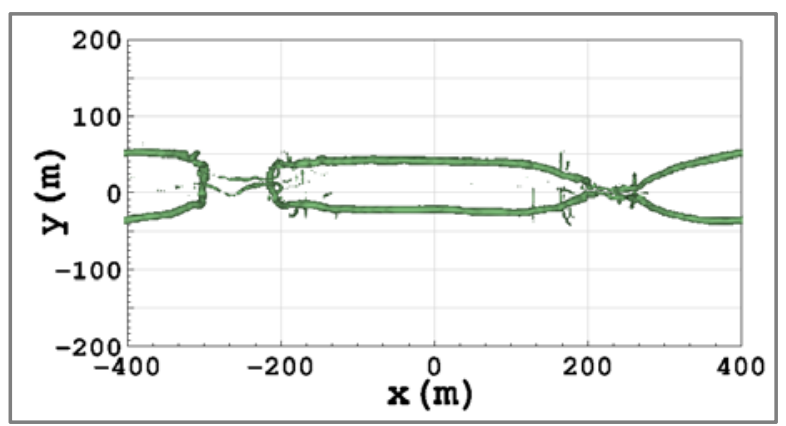

Burnham-Hallock Initialization (120s)

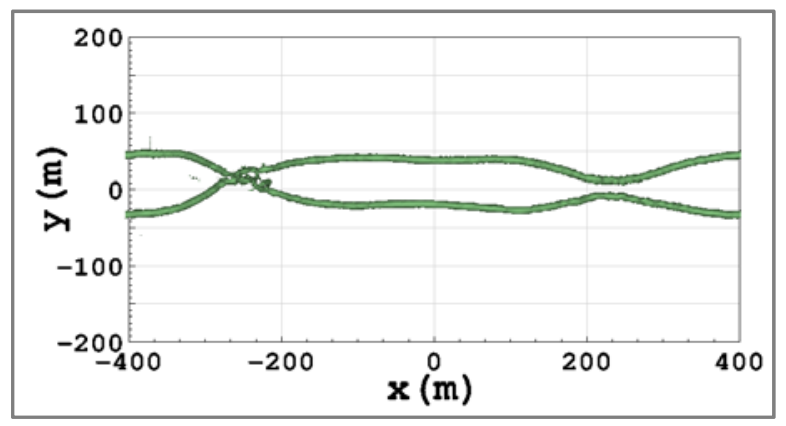

Proctor Initialization (120s)

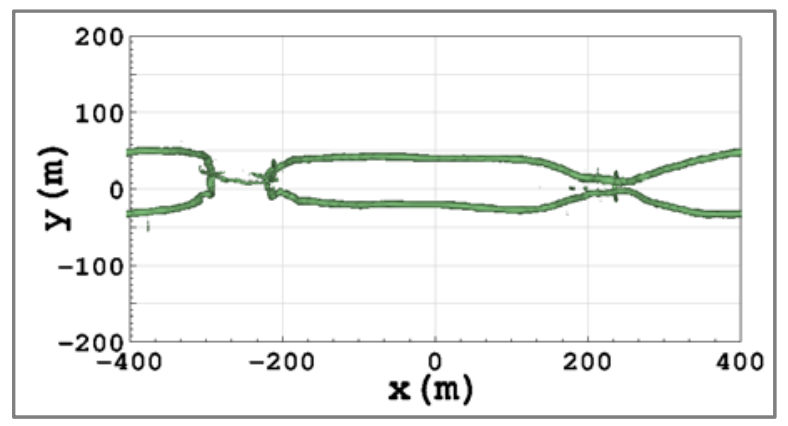

Lamb-Oseen Initialization (120s)

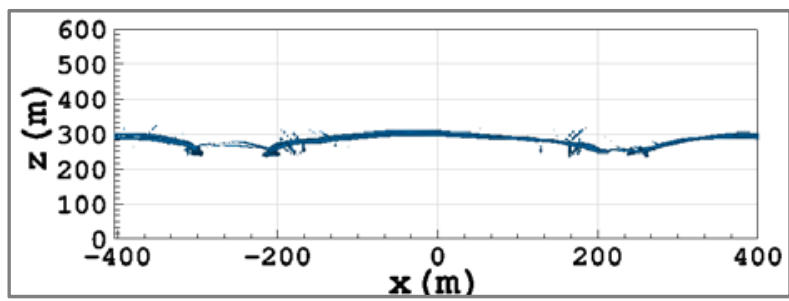

Burnham-Hallock Initialization (120s)

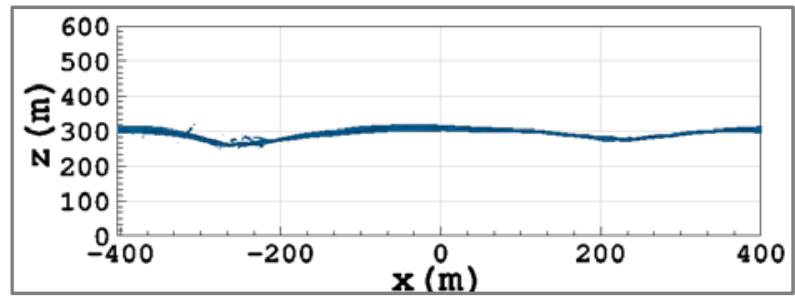

Proctor Initialization (120s)

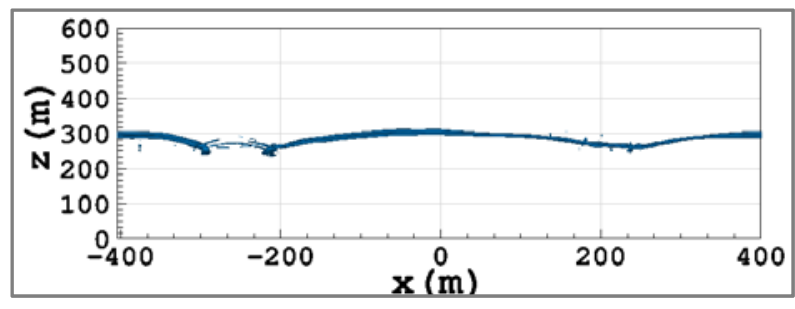

Figure 10. Top and side views of $\lambda_{2}$ isosurface for the three vortex models - Lamb-Oseen (top), BurnhamHallock (middle), and Proctor (bottom). Time $=120 \mathrm{~s} . \Delta x=2 \mathrm{~m}$, and $\Delta y=\Delta z=1.5 \mathrm{~m}$. The vortex core radius, $r_{\mathrm{c}}=4.5 \mathrm{~m} . \mathrm{B} 747 \simeq \Gamma_{0}=565 \mathrm{~m}^{2} / \mathrm{s} . \boldsymbol{b}_{0}=50 \mathrm{~m}$. The vortex pair was initialized at $\left(y_{0}, z_{0}\right)=(0,500) \mathrm{m}$. 
Lamb-0seen Initialization (60s)

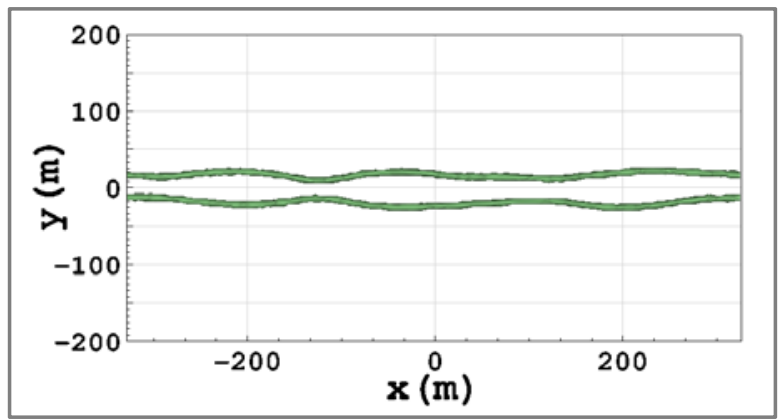

Burnham-Hallock Initialization (60s)

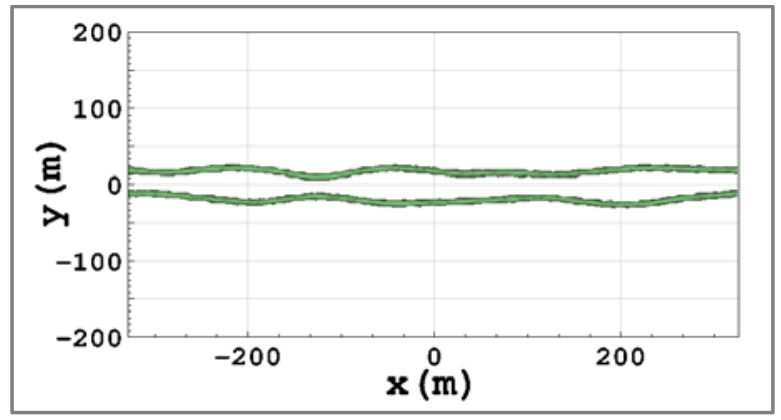

Proctor Initialization (60s)

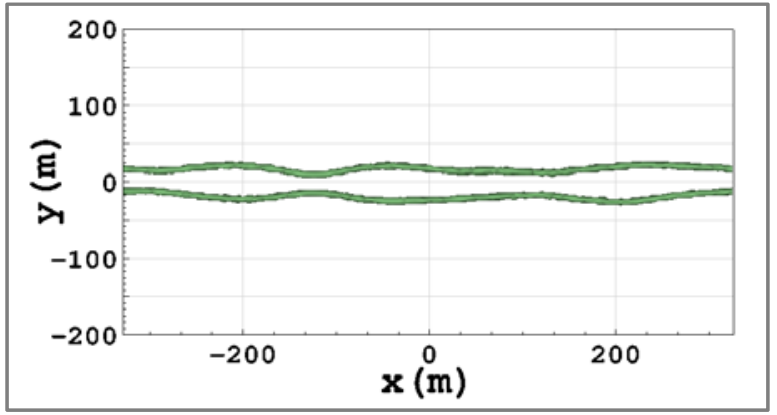

Lamb-Oseen Initialization (120s)

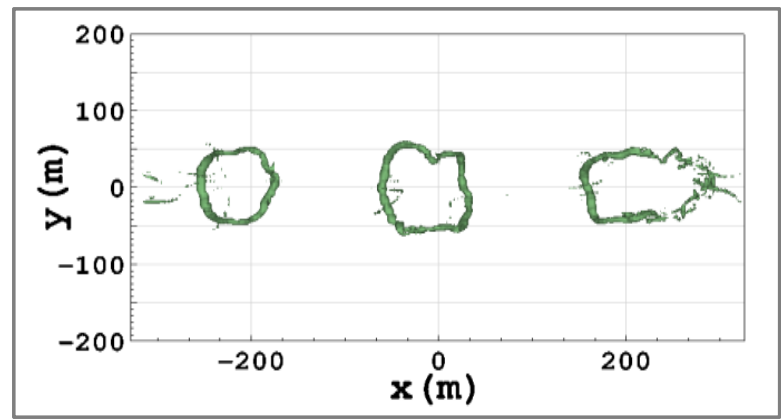

Burnham-Hallock Initialization (120s)

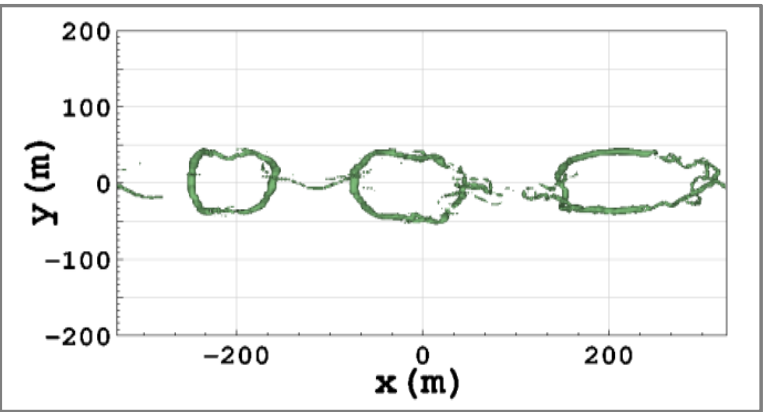

Proctor Initialization (120s)

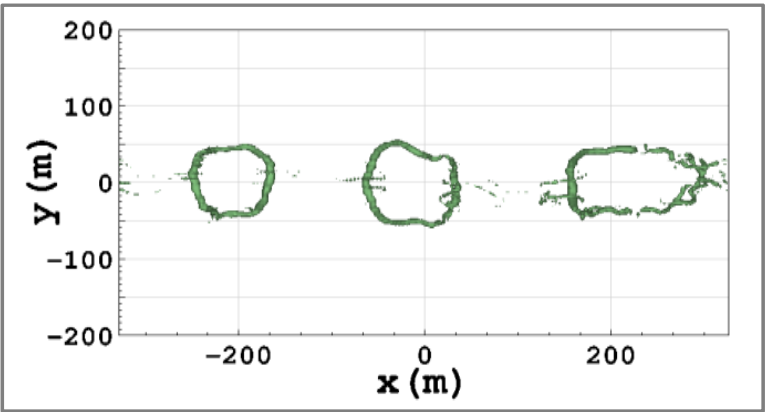

Figure 11. Top views of $\lambda_{2}$ isosurface for the three vortex model initializations - Lamb-Oseen (top), Burnham-Hallock (middle), and Proctor (bottom). Time $=60 \mathrm{~s}$ in the left column and time $=120 \mathrm{~s}$ in the right column. $\Delta x=2 \mathrm{~m}$, and $\Delta y=\Delta z=1.5 \mathrm{~m}$. The vortex core radius, $r_{\mathrm{c}}=3.0 \mathrm{~m}$. B767. $\Gamma_{0}=360 \mathrm{~m}^{2} / \mathrm{s}$. $b_{0}=36 \mathrm{~m}$. The vortex pair was initialized at $\left(y_{0}, z_{0}\right)=(0,142.5) \mathrm{m}$. 


\section{Hazard Circulation}

The circulation, $\Gamma$ is defined as the line integral of the velocity around a closed loop, $C$

$$
\Gamma=\oint_{C} v \cdot d l
$$

where $v$ is the fluid velocity. Using Stokes theorem, the line integral in Eq. (24) can be converted into a surface integral

$$
\Gamma=\oint_{C} v \cdot d l=\int_{S}(\nabla \times v) \cdot d S=\int_{S} \omega \cdot d S .
$$

From Eq. (25), the circulation can be interpreted as the flux of vorticity through the surface, $S$. Figure 12 shows the calculation of $\vec{\omega} \cdot d \vec{S}=\omega_{x} d y d z$ for the Proctor vortex model. The circulation strengths for different integration surface areas are given in Tables 9-10 for two different values of $r_{\mathrm{c}}$. The hazard circulation used in aircraft response models is often $\Gamma_{5-15 \mathrm{~m}}$ (Burnham and Hallock 2013). Compared to the Burnham-Hallock and Proctor models, the values of $\Gamma_{5-15 \mathrm{~m}}$ are much smaller for the Lamb-Oseen model.

Table 9: Circulation $-\mathrm{B} 747-400\left(\Gamma_{0}=565 \mathrm{~m}^{2} / \mathrm{s} \& r_{\mathrm{c}}=3.75 \mathrm{~m}\right)$

\begin{tabular}{|c|c|c|c|}
\hline$\Gamma\left(\mathrm{m}^{2} / \mathrm{s}\right)$ & Lamb-Oseen (LO) & Burnham-Hallock $(\mathrm{BH})$ & Proctor $(\mathrm{FP})$ \\
\hline$\Gamma_{0-40 \mathrm{~m}}$ & 565.00 & 560.07 & 564.48 \\
\hline$\Gamma_{0-15 \mathrm{~m}}$ & 565.00 & 531.75 & 545.20 \\
\hline$\Gamma_{5-15 \mathrm{~m}}$ & 60.20 & 170.20 & 113.83 \\
\hline
\end{tabular}

Table 10: Circulation - B747-400 $\left(\Gamma_{0}=565 \mathrm{~m}^{2} / \mathrm{s} \& r_{\mathrm{c}}=4.5 \mathrm{~m}\right)$

\begin{tabular}{|c|c|c|c|}
\hline$\Gamma\left(\mathrm{m}^{2} / \mathrm{s}\right)$ & Lamb-Oseen (LO) & Burnham-Hallock (BH) & Proctor (FP) \\
\hline$\Gamma_{0-40 \mathrm{~m}}$ & 565.00 & 557.94 & 564.48 \\
\hline$\Gamma_{0-15 \mathrm{~m}}$ & 565.00 & 518.34 & 545.20 \\
\hline$\Gamma_{5-15 \mathrm{~m}}$ & 119.32 & 206.23 & 143.49 \\
\hline
\end{tabular}
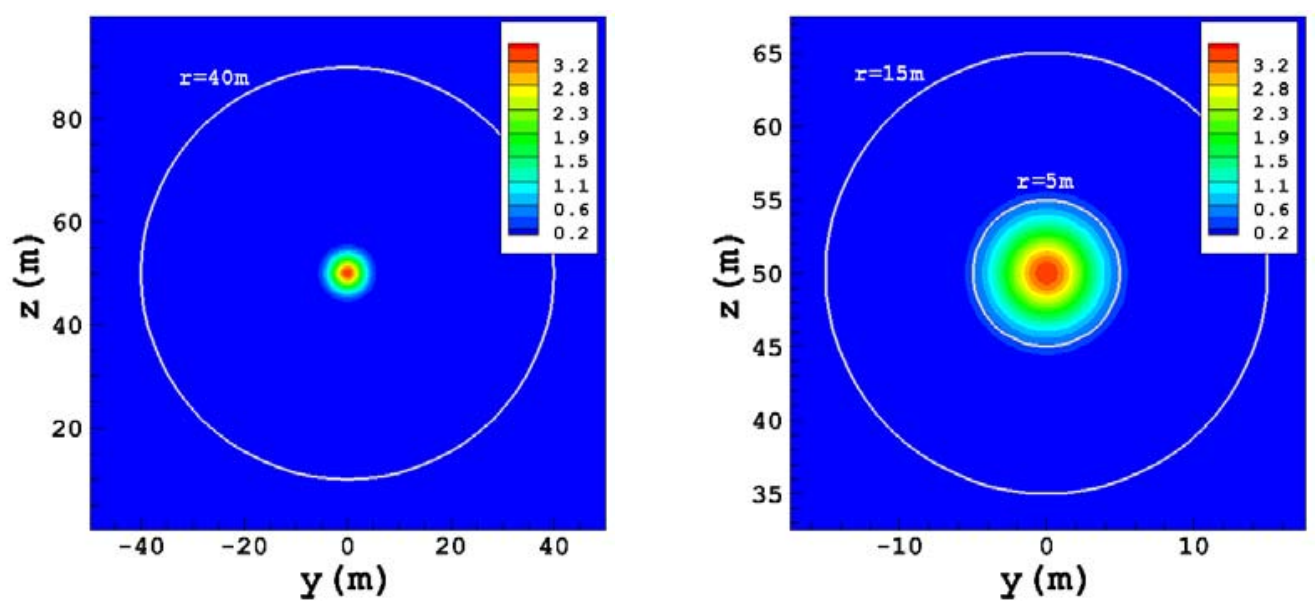

Figure 12. Calculation of $\omega_{X} d y d z\left(\mathrm{~m}^{2} / \mathrm{s}\right)$ for the Proctor model. The left panel shows the entire computational domain, and the right panel shows a zoomed view of the computational domain for $\boldsymbol{r}_{\mathrm{c}}=\mathbf{3 . 7 5 \mathrm { m }}$. 


\section{Lidar Measurements}

\section{A. Velocity Measurement}

For a coherent receiver, the precision with which one can estimate velocity in any given range gate depends on the signal carrier to noise ratio (CNR), the width of the signal spectrum, and the number of independent measurements used to make the estimate. The CNR and the spectral width are influenced by a variety of factors including transmit pulse energy and pulse width, aerosol loading and density variation, wind velocity gradients, and the signal processing method utilized. The number of independent measurements that can be used for making the estimate is typically dictated by sensor requirements.

Periodogram based signal processing is often used in real time systems for making velocity estimates because of computational efficiency. The periodogram is the squared modulus of the discrete Fourier transform of a subset of the discrete samples of the return signal from a single lidar pulse. Each subset of samples of the return signal stream is referred to as a gate (or range gate). The number of discrete samples used (the length of the gate) is up to the user; however, the number of samples is usually chosen based on the range gated window signal coherence time (Eq. 29). Velocity precision is improved (i.e., velocity error is reduced) when the number of independent measurements used to make the velocity estimate is increased. This is achieved by accumulating periodograms from the same gate or adjacent uncorrelated gates (depending on system requirements). Accumulation beats down shot noise fluctuations in the signal spectral estimate including fluctuations caused by speckle, and increases the signal detectivity.

Figure 13 contains two example normalized periodograms: a single realization on the left and the average of 50 independent realizations on the right. For this data the central bin, Bin 0 , represents no wind or a velocity of $0 \mathrm{~m} / \mathrm{s}$. The spectral resolution of this data is $1.5625 \mathrm{MHz}$ per frequency bin and the optical wavelength is $\lambda=2 \mu \mathrm{m}$. A velocity estimate is made by converting the measured Doppler shift to velocity using:

$$
V_{r}=\frac{-\lambda f_{D}}{2} .
$$

The peak in the spectrum observed at Bin 2 corresponds to a Doppler shift of $f_{D}=3.125 \mathrm{MHz}$ or a velocity of $-3.125 \mathrm{~m} / \mathrm{s}$ (winds towards the lidar). To improve accuracy of the estimate, zero padding or a parabolic fit are used to better determine the peak location. The actual Doppler shift in this example is $3.5 \mathrm{MHz}$ corresponding to fractional Bin 2.24 and a velocity of $-3.5 \mathrm{~m} / \mathrm{s}$.
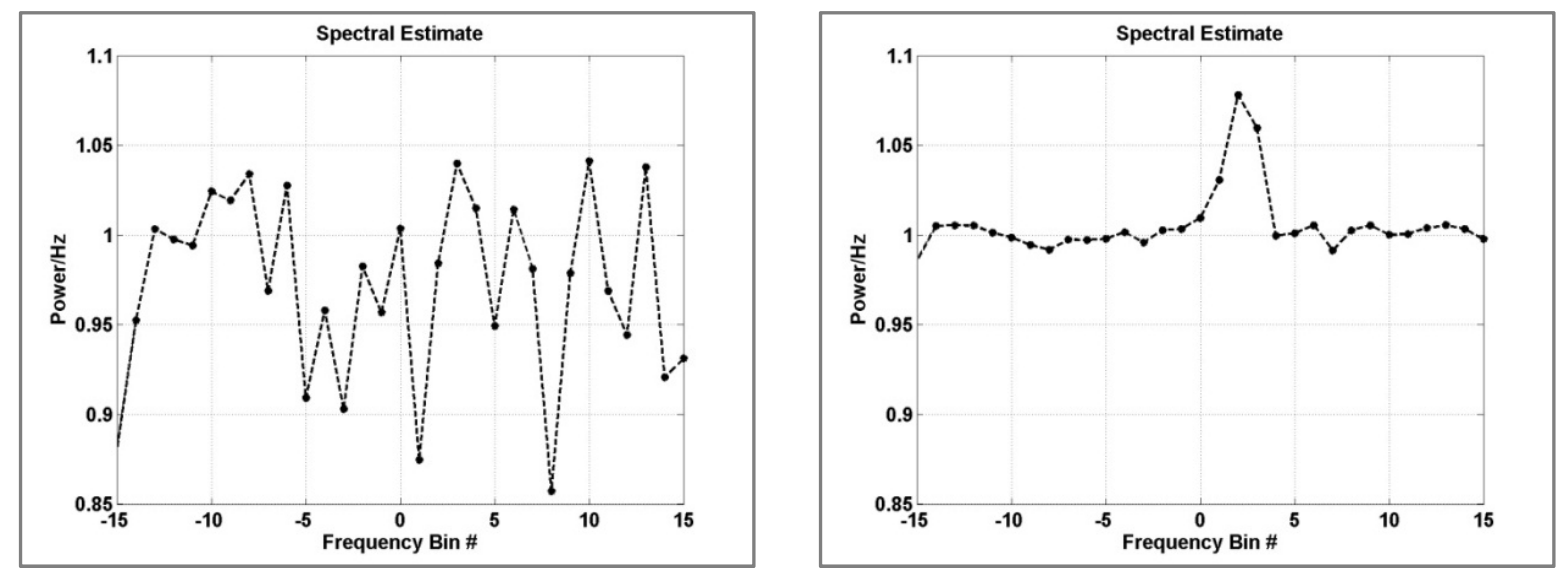

Figure 13: Normalized single realization periodogram (left) and 50 pulse accumulation (right). Spectral resolution of $1.5625 \mathrm{MHz}$, wavelength of $2 \mu \mathrm{m}$ and line of sight wind speed of $3.5 \mathrm{~m} / \mathrm{s}$ towards the lidar.

\section{A.1 CNR}

CNR is defined as the height of the detected signal spectral peak above a unit noise floor and can be expressed as (Henderson et al. 2005; Targ et al. 1991):

$$
C N R=\frac{\eta_{s}^{\prime}}{F_{h}} \frac{E}{h v} T^{2} \beta \frac{C}{2 B} \frac{A_{r}}{R^{2}} \frac{1}{S R F} .
$$


where $\eta_{s}^{\prime}$ is the far field system efficiency of the lidar and in the absence of refractive turbulence (in well-designed truncated-beam lidar systems $\eta_{s}^{\prime}$ is in the range of $\left.10-15 \%\right), F_{h}$ is the excess noise factor above the shot noise limit (in well-designed coherent receivers $\left.F_{h}>0.9\right)$, E (J) is the transmitter pulse energy, $h=6.626 \times 10^{-34}(\mathrm{~J} \cdot s)$ is Planck's constant, $v(\mathrm{~Hz})$ is the optical frequency, $T$ is the one-way path dependent atmospheric transmission, $\beta\left(\mathrm{m}^{-1} \mathrm{sr}^{-1}\right)$ is the path dependent backscatter coefficient, $c$ is the speed of light, $B(\mathrm{~Hz})$ is the receiver noise equivalent bandwidth, $A_{r}\left(\mathrm{~m}^{2}\right)$ is the area of the receiver aperture, $R(\mathrm{~m})$ is range, and SRF is the signal reduction factor representing inefficiencies associated with diffraction, focus, refractive turbulence, and beam truncation.

For a Gaussian signal spectrum the "optimal" operating point in terms of the best trade of range resolution and CNR is a matched Gaussian integration window, which gives a noise equivalent bandwidth of (Jacob et al. 2009):

$$
B_{n}=1 / \tau_{c g},
$$

where $\tau_{c g}$ is Goodman's coherence time for the received signal field defined as (Goodman 1984):

$$
\tau_{c g}=\int_{-\infty}^{\infty}|\gamma(\tau)|^{2} d \tau
$$

where $\gamma(\tau)$ is the complex autocorrelation function of the received signal. For a Gaussian signal spectrum and a matched Gaussian integration window, Goodman's coherence time is related to the transmit pulse full width at half maximum (FWHM), $\Delta T$, by

$$
\tau_{c g}=\sqrt{\frac{\pi}{2 \ln 2}} \Delta T \sim 1.505 \Delta T .
$$

Of course, one is free to choose any shape and duration for the signal processing integration window. Increasing the duration of the window increases CNR up to the intrinsic limit at the cost or range resolution. It is customary, because of its simplicity, to use a rectangular integration window. Doing so results in negligible difference in the CNR when compared to the matched Gaussian window of the same duration.

\section{A.2 Velocity Precision}

The minimum velocity error variance that can be achieved with an unbiased optimal estimator can be expressed in terms of the narrowband CNR as (Henderson et al. 2005; Van Trees 1968; Doviak and Zrnic 1984):

$$
\operatorname{var}\left[V_{r}\right] \geq\left(\frac{\lambda}{2}\right)^{2} \frac{2 \delta v^{2}}{M}\left[\frac{1}{C N R}+\frac{1}{C N R^{2}}+\frac{1}{4}\right],
$$

where $\delta v(\mathrm{~Hz})$ is the windowed signal spectrum RMS width, $M$ is the number of independent incoherent averages (accumulated periodograms) used to make the estimate, and the third term in Eq. (31) is a saturation term that accounts for speckle. Assuming a transform limited Gaussian pulse, matched integration window, uniform backscatter, and no wind turbulence, the RMS spectral width is related to the pulse FWHM as:

$$
\delta v=\frac{\sqrt{\ln 2}}{\sqrt{2} \pi \Delta T} .
$$

\section{A.3 Detectivity}

To make a velocity estimate, one must be able to distinguish the peak of the spectrum from the fluctuations in the noise background. The detectivity is a measure of the height of signal spectrum peak relative to the fluctuation in the background (i.e., noise floor). The detectivity, or figure of merit (FOM), is defined as the ratio of the expected signal peak level to the standard deviation of the noise floor (Henderson et al. 2005). As mentioned previously, the CNR is the height of the signal peak above the normalized to unity noise floor. The normalized noise floor has a standard deviation of $1 / \sqrt{M}$ the detectivity is then:

$$
F O M=\sqrt{M} C N R .
$$


The FOM speaks to the statistics of the power in any spectral bin and is not a function of any particular system parameter. Hence, it is a good metric for defining the maximum working range of a lidar system and useful when comparing the performance of one system to another. Empirical results indicate that a FOM 2 results in approximately half of the estimates being good estimates and half being anomalies (or false detections due to noise). The range corresponding to $F O M \sim 2$ represents the effective working range of the coherent system (Henderson et al. 2005).

\section{B. Wake Vortex Measurement}

The wake vortex measurement problem differs from the typical wind measurement problem described in the previous section in that the aircraft wake velocity flow field contains significant velocity gradients over lengths typically shorter than the pulse length. Velocity gradients increase the spectral content of the return signal (i.e., increase the signal spectrum RMS width) and reduce the signal spectral peak (i.e., reduce the CNR). By Eq. (31) both of these effects work towards reducing the precision of a velocity measurement. Moreover, the velocity gradients observed in a wake velocity field typically generate non-Gaussian spectral profiles. Making a velocity estimate on wake influenced spectral measurement only estimates the strongest weighted average velocity component over the length of the range gate and provides no information of the velocity distribution that produced the measured spectra. The addition of an estimate of the spectral width provides some information on the velocity distribution, but the actual velocity profile of the wake along a given line of sight is not recoverable from these two estimates.

If one cannot better resolve the velocity profile by using a shorter pulse (or through more advanced signal processing techniques) then a better approach is to more effectively utilize the spectral information that is available. For example, for a given spectral estimate, instead of only estimating the peak velocity one can also estimate the minimum and maximum velocities above some noise threshold. This information provides additional information about the shape of the spectrum. This information combined with knowledge of the system and how the signal processing influences the spectra (the effects of the pulse width, range gating, pulse averaging, scan rate, spectral resolution, etc.,) can then be used to estimate vortex position and circulation strength (Banakh and Smalikho 2013).

Another approach used for wake vortex estimation is to compare the lidar measured spectra against model spectra based on a vortex flow model (Hannon and Thompson 1994). For transverse viewing of aircraft wakes, model spectra are constructed based on a vortex tangential velocity model such as those described earlier in this paper. The model spectra take into account all the system and signal processing influences that combine to form the spectral estimate (Frehlich and Sharman 2005).

\section{B.1 Transverse Viewing Geometry}

The transvers viewing geometry for wake measurements is shown in Figure 14. The lidar is positioned transverse to the direction of flight and scans continuously over some portion of the plane perpendicular to the axial component of the vortex velocity flow field. As the aircraft moves through the scan, plan aerosols in the atmosphere become entrained in the wake. The energy from each transmitted pulse scatters off the aerosols that are positioned along the lidar line of sight. The motion of the aerosols Doppler shifts the energy scattered back to the receiver affecting the spectral content of the return signal.

The lidar can only sense that component of motion along its line of sight. For transverse viewing, this means a component of the tangential flow field can be sensed. However, what the lidar actually measures will depend on the system characteristics and the signal processing and will not simply be equal to the dot product of the tangential velocity and the lidar line of sight vector.

In the results that follow, a lidar system with an optical wavelength of $\lambda=2 \mu \mathrm{m}$ and a sample frequency $f_{\mathrm{s}}=100 \mathrm{MHz}$, which equates to a velocity bandwidth of $\Delta f= \pm 25 \mathrm{~m} / \mathrm{s}$ with real sampling, is used and for the signal processing a range gate length of $\Delta R \sim 1.5 \Delta T$, a spectral resolution of $\Delta f=1.5625 \mathrm{MHz}$ and spectral peak detection with parabolic fit are used. All signals are ideal (i.e., shot noise and speckle free) for illustrative convenience.

For simplicity in this discussion, a single vortex with clockwise rotation will be used as shown in Figure 15 . The vortex will be that of the B747-400 with $\Gamma_{0}=565 \mathrm{~m}^{2} / \mathrm{s}$ and $r_{\mathrm{c}}=3.75 \mathrm{~m}$. It will be centered at a range of $\mathrm{R}=1.023 \mathrm{~km}$ and at an elevation angle of $\phi=0$ degrees. Results will be shown for a single range gate centered at $1.023 \mathrm{~km}$ and for elevations ranging from $\phi=-3.01$ to +3.01 degrees with line of sight increments of $\Delta \phi=0.07$ degrees as depicted in the left panel of Figure 15. These choices conveniently align one line of sight on each side of the vortex core with the peak tangential velocity located at one core radius. The elevation span corresponds to approximately $54 \mathrm{~m}$ along the vortex radial. 


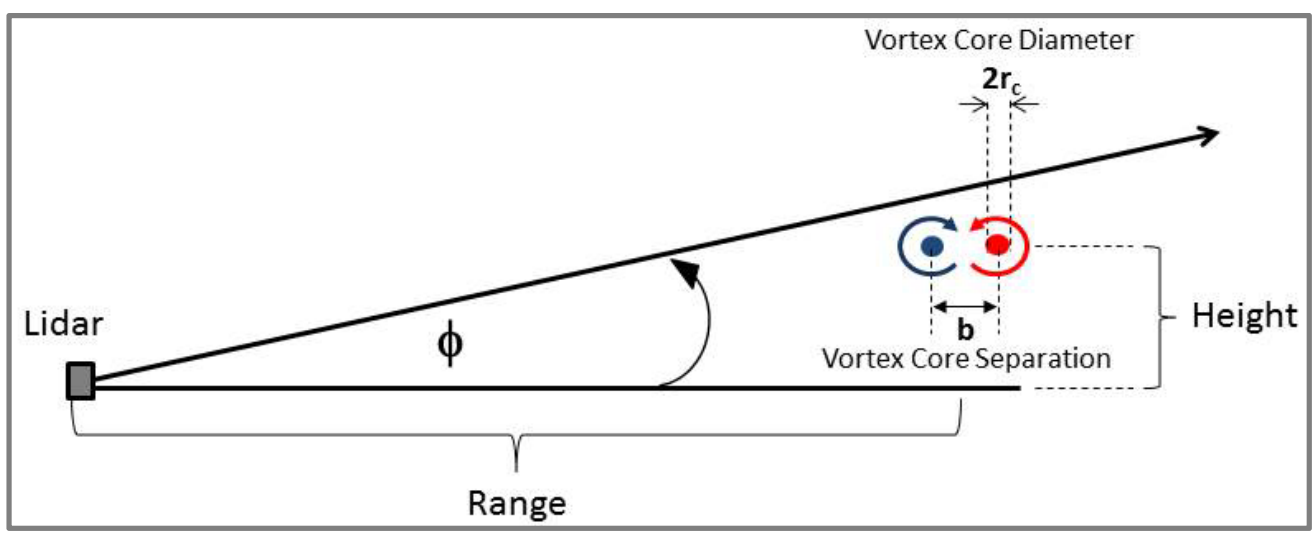

Figure 14: Transverse viewing geometry.
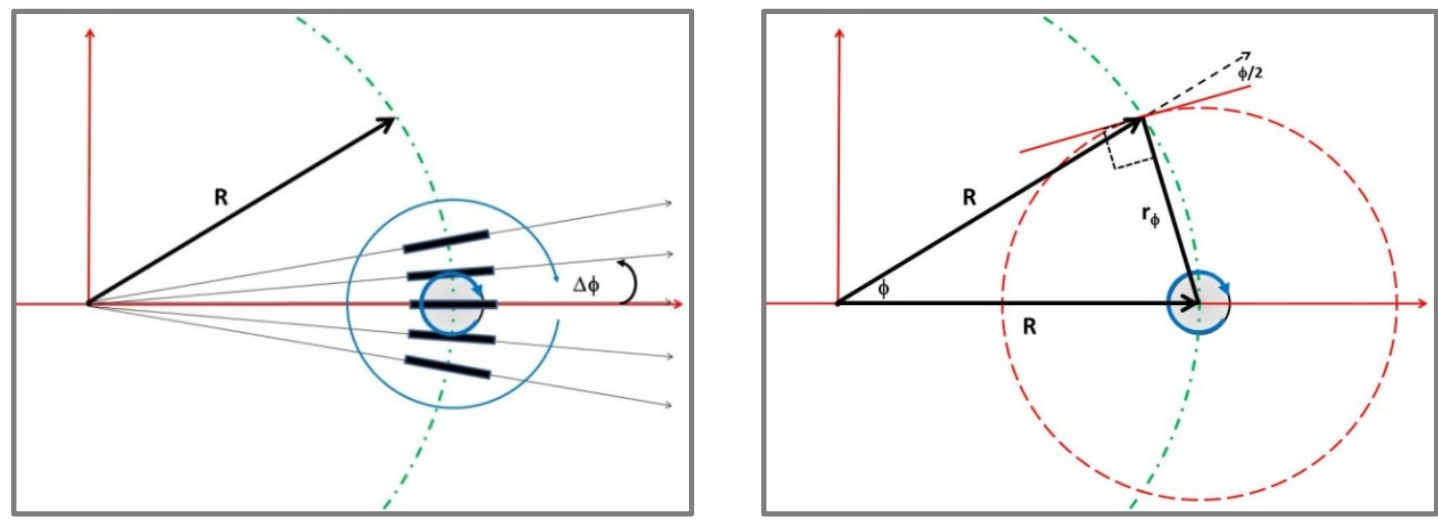

Figure 15: Single vortex geometry used in the analysis. Left panel: range gates at fixed range. Right Panel: Geometry for determining radial distance to center of range gate.

From the geometry shown in the right panel of Figure 15 the radius from the center of the vortex to the center of each range gate can be shown to be given by:

$$
r_{\phi}=R \frac{\sin (\phi)}{\cos (\phi / 2)} .
$$

The model velocity along the lidar line of sight at the center of each range gate is then:

$$
v_{r}=v_{\theta}\left(r_{\phi}\right) \cos (\phi / 2),
$$

where $v_{\theta}$ is the model tangential velocity.

\section{B.2 Model Spectra and Measured Velocity Profiles}

The normalized model signal spectra (Frehlich and Sharman 2005) as a function of elevation angle for the B747400 with $\Gamma_{0}=565 \mathrm{~m}^{2} / \mathrm{s}$ and $r_{\mathrm{c}}=3.75 \mathrm{~m}$ as a function of elevation are shown in Figure 16 for pulse widths of $\Delta T=400 \mathrm{~ns}$ (top row) and $\Delta T=100 \mathrm{~ns}$ (bottom row) for the Burnham-Hallock (left), Lamb-Oseen (center) and Proctor (right) wake vortex tangential velocity models described earlier. Inspection of the spectra reveal variations in the spectral signatures in the vicinity of the core as a function of frequency and elevation when comparing the signatures associated with different vortex models (conceptually similar to what is shown in Figure 5). 

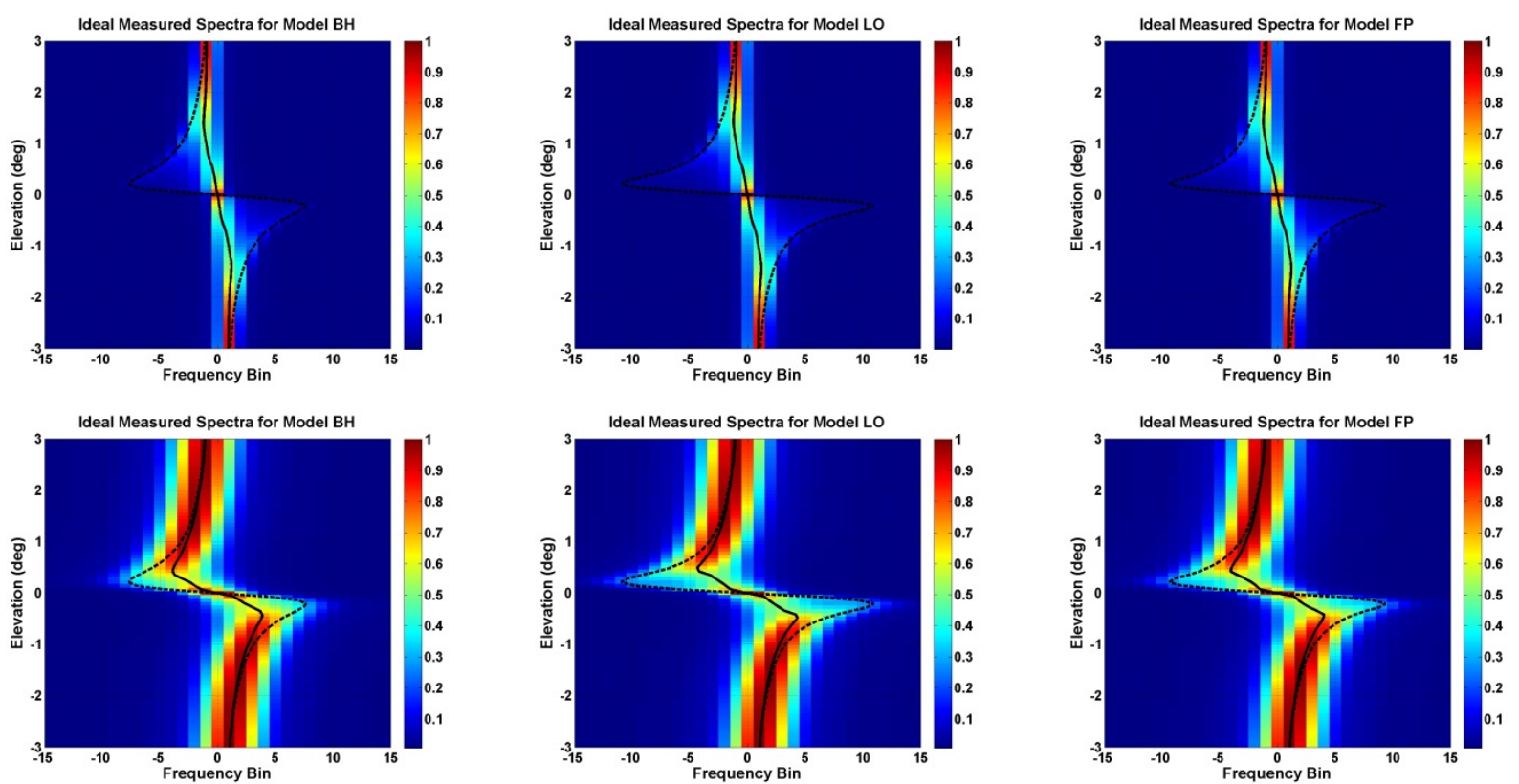

Figure 16: Normalized model signal spectra for $B 747-400$ with $\Gamma_{0}=565 \mathrm{~m}^{2} / \mathrm{s}$ for pulse widths of $\Delta T=400 \mathrm{~ns}$ (top row) and $\Delta T=100 \mathrm{~ns}$ (bottom row) for the Burnham-Hallock (left), Lamb-Oseen (center), and Proctor (right) wake vortex tangential velocity models.

The spectral peak (fitted) for each elevation is indicated by the solid black curve in each plot, and for comparison, the model velocity in frequency space is indicated by the dashed black curve. As can be seen, the measured spectral peak and the model spectral peak values differ significantly; however, less so for the shorter pulse width.

The three model velocity profiles (dashed curve) and the resulting lidar measured velocity profiles (solid curve) are compared in Figure 17 for pulse widths $\Delta T=400 \mathrm{~ns}$ (left column) and $\Delta T=100 \mathrm{~ns}$ (right column) using the spectral peak values from Figure 16 with Eq. (26). As can be seen the lidar measured velocity profiles are highly filtered versions of the model profiles and show negligible difference from model to model.
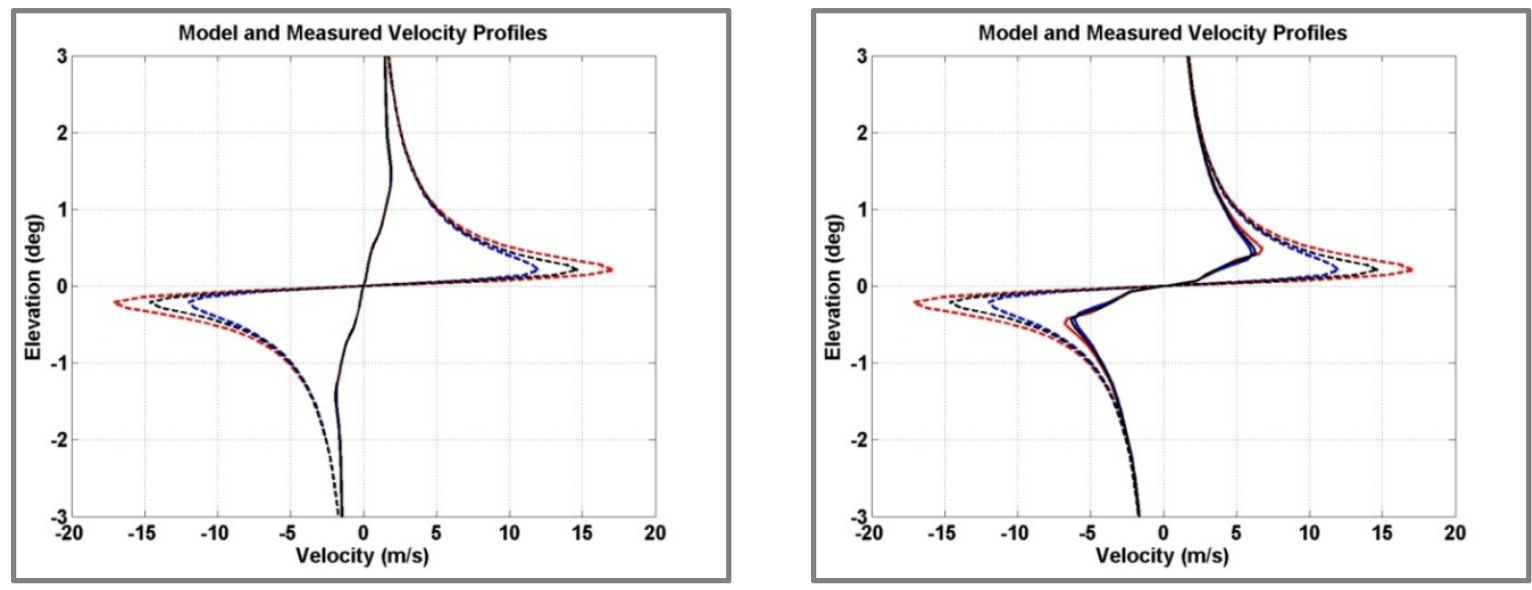

Figure 17: Comparison of model and measured velocity profiles. Model profiles (solid) and measured profiles (dashed) with Burnham-Hallock (blue), Lamb-Oseen (red), and Proctor (black).

The Burnham-Hallock model and measured velocity profiles for circulation strengths of $\Gamma_{0}=465 \mathrm{~m}^{2} / \mathrm{s}$ (black), $\Gamma_{0}=565 \mathrm{~m}^{2} / \mathrm{s}$ (blue) and $\Gamma_{0}=665 \mathrm{~m}^{2} / \mathrm{s}$ are shown in Figure 18 for pulse widths $\Delta T=400 \mathrm{~ns}$ (left column) and 
$\Delta T=100 \mathrm{~ns}$ (right column). For the longer pulse, there are only minute differences in the measured velocity profiles, while for the shorter pulse differences in the measured velocities are more apparent. As one might guess, using the measured velocity profile by itself to make an estimate of the circulation strength in noisy conditions is not the best approach because of the system response filtering that occurs.
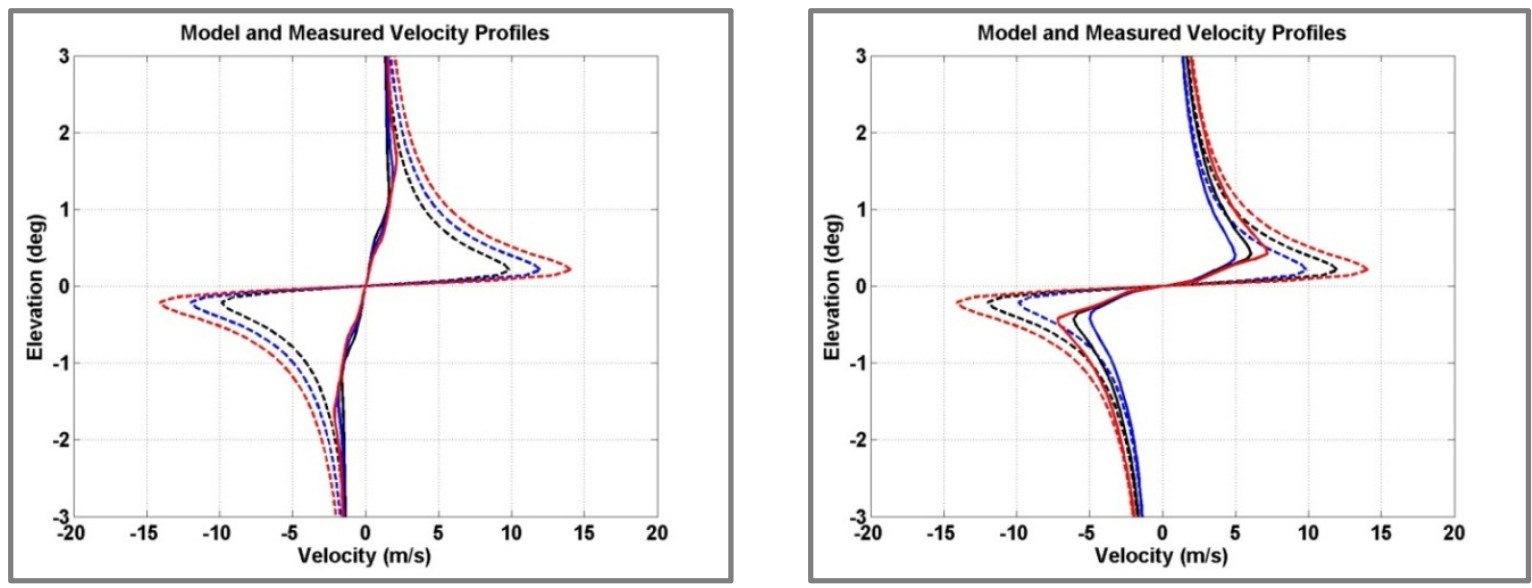

Figure 18: Burnham-Hallock model (solid) and measured (dashed) velocity profiles for a B747-400 single vortex for a pulse width of $\Delta T=400 \mathrm{~ns}$ and $\Gamma_{0}=465 \mathrm{~m}^{2} / \mathrm{s}$ (black), $\Gamma_{0}=565 \mathrm{~m}^{2} / \mathrm{s}$ (blue) and $\Gamma_{0}=665 \mathrm{~m}^{2} / \mathrm{s}$ (red).

A better approach is one based on a more detailed analysis of the spectral content of the return signal. One can define velocity envelopes by thresholding the measured signal spectra above some level to estimate minimum and maximum velocities to better define the measured velocity distribution for more accurate estimate of the circulation strength (Banakh and Smalikho 2013). In another approach, one can compare directly model spectra of different circulation strengths with the measured spectral distribution (Hannon and Thompson 1994). Figure 19-20 show the model spectra for circulation strengths $\Gamma_{0}=465 \mathrm{~m}^{2} / \mathrm{s}$ (left), $\Gamma_{0}=565 \mathrm{~m}^{2} / \mathrm{s}$ (center), and $\Gamma_{0}=665 \mathrm{~m}^{2} / \mathrm{s}$ (right) for pulse widths $\Delta T=400 \mathrm{~ns}$ and $\Delta T=100 \mathrm{~ns}$ respectively. The model velocity profile and the measured velocity profile are also shown in frequency space by the black dashed and solid curves respectively.

Close inspection of the spectra shown below reveal multiple observable variations in the spectral signatures as a function of frequency and as a function of elevation when comparing the signatures associated with different circulation strengths. Just as multiple independent measurements help improve the precision of a velocity estimate, using all of the spectral data will help improve the precision of a wake circulation estimate; however, performance will rely on the accuracy of the model velocity flow with actual flows measured in the field. With noisy signals, these comparisons are made using techniques that take into consideration the spectral statistics of the signal and noise and assess how well the spectral model fits the measured signature (Frehlich and Sharman 2005).
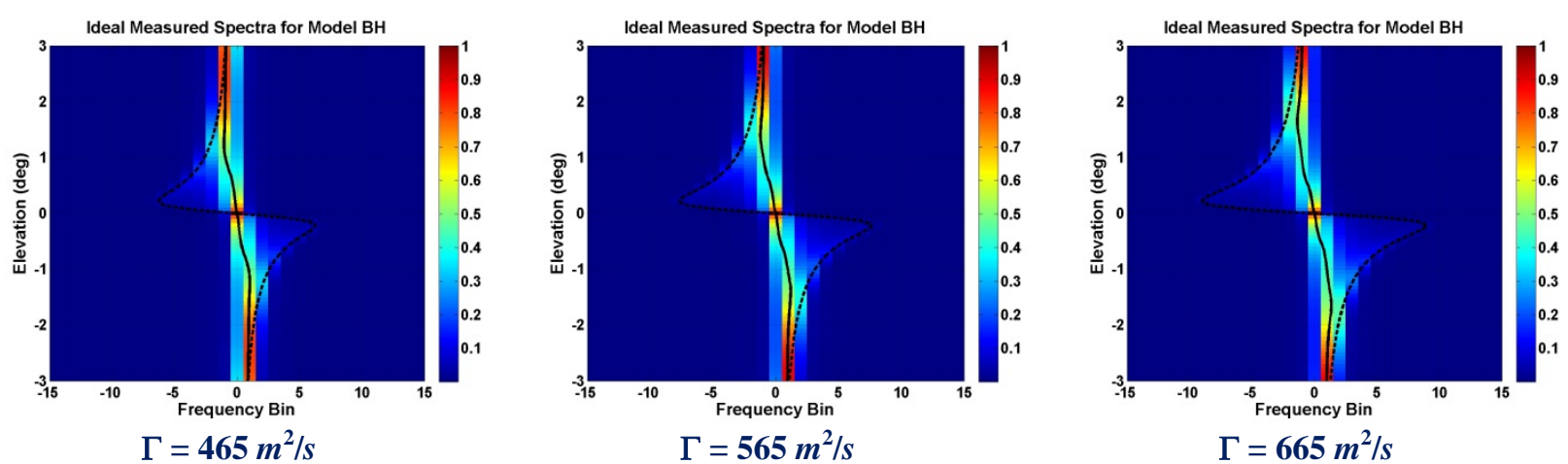

Figure 19: Burnham-Hallock Model signal spectra for a B747-400 single vortex for a pulse width of $\Delta T=400 \mathrm{~ns}$ and $\Gamma_{0}=465 \mathrm{~m}^{2} / \mathrm{s}$ (left), $\Gamma_{0}=565 \mathrm{~m}^{2} / \mathrm{s}$ (center) and $\Gamma_{0}=665 \mathrm{~m}^{2} / \mathrm{s}$ (right). 

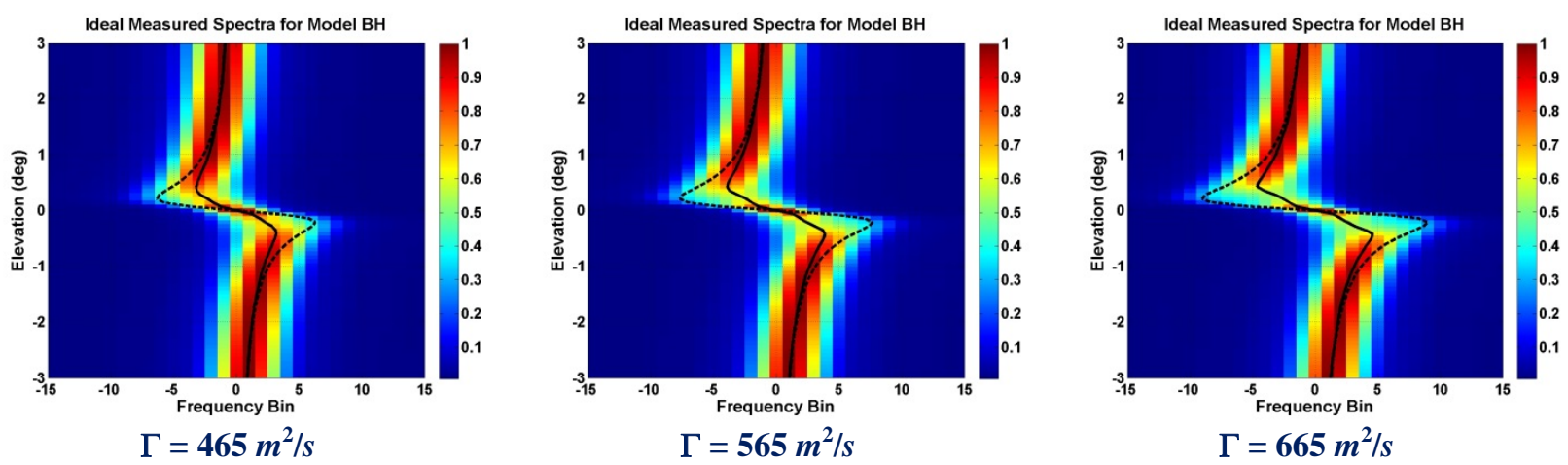

Figure 20: Burnham-Hallock Model signal spectra for a B747-400 single vortex for a pulse width of $\Delta T=100 \mathrm{~ns}$ and $\Gamma_{0}=465 \mathrm{~m}^{2} / \mathrm{s}$ (left), $\Gamma_{0}=565 \mathrm{~m}^{2} / \mathrm{s}$ (center) and $\Gamma_{0}=665 \mathrm{~m}^{2} / \mathrm{s}$ (right).

\section{Summary}

In this paper, the properties of three idealized aircraft wake vortex models were described in detail. The effect of vortex core radius size on tangential velocities was discussed. The Lamb-Oseen model predicts the highest peak tangential velocity and shows the largest variation in peak tangential velocity as a function of vortex core radius. The Burnham-Hallock model has the smallest peak tangential velocity, and the Proctor model exhibits the smallest variation in peak tangential velocity with change in core radius size. The tangential velocity approaches zero at the vortex center and at large distances from the vortex center for all three models. The tangential velocity attains its maximum value at the vortex core radius. The vortex circulation is zero at the vortex center for all three models and approaches $\Gamma_{0}$ at $r \rightarrow \infty$. All three models satisfy the divergence property, and the vortex region definition is similar in terms of vorticity and $\lambda_{2}$.

The field maximums and minimums in the two-dimensional Euler simulations were larger for Lamb-Oseen initializations compared to the other two models. In the three-dimensional TASS simulations, the theoretical value of time-to-link, $T_{\mathrm{L}}$ was used for comparing the simulations. Faster vortex linking occurred with Lamb-Oseen initializations, whereas the $T_{\mathrm{L}}$ values predicted by Burnham-Hallock initializations were closest to the theoretical value. The hazard circulations, $\Gamma_{5-15 \mathrm{~m}}$ were compared for two vortex core sizes. Compared to the Burnham-Hallock and Proctor models, the values of $\Gamma_{5-15 \mathrm{~m}}$ were much smaller for the Lamb-Oseen model. An aircraft response model for wake encounter based on the Lamb-Oseen vortex will underestimate the encounter hazard.

A brief introduction to the wake measurement problem was presented highlighting the differences between velocity estimation and wake estimation. Coherent pulsed lidar is an effective tool for detecting and measuring aircraft wake vortices. Wake measurements rely on close scrutiny of the spectral content of the return signal. In order to accurately estimate vortex parameters such as circulation strength, position, and core size, a complete understanding of how the lidar system parameters and signal processing affect the spectral content of the measured wake signature are required. Wake processing of return signals from long pulse lidars are expected to be less sensitive to the choice of vortex model used due to a high degree of system response filtering that occurs. Wake processing for shorter pulse systems may be more affected by model choice.

\section{Appendix A: Navier-Stokes Solution of Lamb-Oseen Vortex}

The Lamb-Oseen vortex model is derived in this appendix. The momentum equation is given by

$$
\frac{\partial \overrightarrow{\mathrm{V}}}{\partial \mathrm{t}}+(\vec{V} \cdot \vec{\nabla}) \vec{V}-\vec{F}+\frac{1}{\rho} \vec{\nabla} p-v \nabla^{2} \vec{V}=0
$$

Taking the curl of the momentum equation (A.1)

$$
\vec{\nabla} \times\left(\frac{\partial \vec{V}}{\partial \mathrm{t}}+(\vec{V} \cdot \vec{\nabla}) \vec{V}-\vec{F}+\frac{1}{\rho} \vec{\nabla} p-v \nabla^{2} \vec{V}=0\right) .
$$

From vector algebra, $\vec{\nabla} \times \vec{\nabla} p=0$, for a conservative field, $\vec{\nabla} \times \vec{F}=0$, and, 


$$
\vec{\nabla} \times(\vec{V} \cdot \vec{\nabla}) \vec{V}=\vec{\nabla} \times\left(\frac{1}{2} \vec{\nabla}(\vec{V} \cdot \vec{V})-\vec{V} \times \vec{\omega}\right) \Rightarrow \vec{\nabla} \times(\vec{V} \cdot \vec{\nabla}) \vec{V}=(\vec{V} \cdot \vec{\nabla}) \vec{\omega}-(\vec{\omega} \cdot \vec{\nabla}) \vec{V}
$$

Eq. (A.1) simplifies to

$$
\frac{\partial \vec{\omega}}{\partial \mathrm{t}}+(\vec{V} \cdot \vec{\nabla}) \vec{\omega}-(\vec{\omega} \cdot \vec{\nabla}) \vec{V}=v \nabla^{2} \vec{\omega}
$$

For two-dimensional flows, Eq. (A.3) can be re-written as

$$
\frac{\partial \omega}{\partial \mathrm{t}}+(\vec{V} \cdot \vec{\nabla}) \omega=v \nabla^{2} \omega
$$

Ignoring non-linear terms in Eq. (A.4)

$$
\frac{\partial \omega}{\partial \mathrm{t}}=v \nabla^{2} \omega
$$

Eq. (A.5) can be re-written in cylindrical coordinates as

$$
r \frac{\partial \omega}{\partial \mathrm{t}}=v \frac{\partial^{2} \omega}{\partial r^{2}}-\frac{v}{r} \frac{\partial \omega}{\partial r}
$$

Using the similarity solution,

$$
\omega(r, t)=f(\eta), \text { where } \eta=\frac{r}{\sqrt{v t}}
$$

Finding the terms in Eq. (A.6),

$$
\begin{aligned}
& \frac{\partial \omega}{\partial t}=\frac{\partial \omega}{\partial \eta} \frac{\partial \eta}{\partial t}=f^{\prime} \frac{-r t^{-3} / 2}{2 \sqrt{v}} \\
& \frac{\partial \omega}{\partial r}=\frac{\partial \omega}{\partial \eta} \frac{\partial \eta}{\partial r}=f^{\prime} \frac{1}{\sqrt{v t}} . \\
& \frac{\partial^{2} \omega}{\partial r^{2}}=f^{\prime \prime} \frac{1}{\sqrt{v t}}
\end{aligned}
$$

Substituting Eq. (A.8) into Eq. (A.6),

$$
f^{\prime \prime}+\left(\frac{\eta}{2}-\frac{1}{\eta}\right) f^{\prime}=0
$$

The solution of Eq. (A.9) is given by:

$$
f=A \exp \left(\frac{-\eta^{2}}{4}\right)+B
$$

Substituting Eq. (A.10) into Eq. (A.7),

$$
\omega(r, t)=A \exp \left(\frac{-r^{2}}{4 v t}\right)+B .
$$


Circulation, $\Gamma$ in terms of vorticity is given by

$$
\Gamma=\pi r^{2} \omega
$$

Substitute $\omega(r, t)$ from Eq. (A.11) into Eq. (A.12)

$$
\Gamma(r, t)=A \pi r^{2} \exp \left(\frac{-r^{2}}{4 v t}\right)+B \pi r^{2}
$$

At $t=0, \Gamma(r, 0)=B \pi r^{2}=\Gamma_{0}$ and at $r=0, \Gamma(0, t)=A+B=0$

$$
A=-B=\frac{-\Gamma_{0}}{\pi r^{2}}
$$

Substituting the values of $A$ and $B$ from Eq. (A.14) into Eq. (A.13),

$$
\Gamma(r, t)=\Gamma_{0}\left(-\exp \left(\frac{-r^{2}}{4 v t}\right)+1\right) .
$$

Eq. (A.12) can also be written in terms of tangential velocity

$$
\Gamma=2 \pi r v_{\theta} .
$$

Substituting $\Gamma$ from Eq. (A.15) into Eq. (A.16)

$$
v_{\theta}(r, t)=\frac{\Gamma_{0}}{2 \pi r}\left(-\exp \left(\frac{-r^{2}}{4 v t}\right)+1\right) .
$$

The tangential velocity, $v_{\theta}$ is maximum at $r=r_{\mathrm{c}}$. Differentiating Eq. (A.17) w.r.t $r$ and setting it to zero,

$$
\frac{\Gamma_{0}}{2 \pi r_{c}^{2}}\left(-1+\exp \left(\frac{-r_{c}^{2}}{4 v t}\right)+\frac{2}{4} \frac{r_{c}^{2}}{v t} \exp \left(\frac{-r_{C}^{2}}{4 v t}\right)\right)=0 .
$$

Simplifying Eq. (A.18),

$$
\exp \left(\frac{-r_{c}^{2}}{4 v t}\right)\left(1+2 \frac{r_{c}^{2}}{4 v t}\right)=1
$$

Solving Eq. (A.19) for $r_{c}^{2} / 4 v t$,

$$
\frac{r_{c}^{2}}{4 v t} \cong 1.256 \Rightarrow \frac{1}{4 v t}=\frac{1.256}{r_{c}^{2}}
$$

Substituting $\frac{1}{4 v t}=\frac{1.256}{r_{c}^{2}}$ from Eq. (A.20) into Eq. (A.17),

$$
v(r, t)=\frac{\Gamma_{0}}{2 \pi r}\left(1-\exp \left(-1.256 \frac{r^{2}}{r_{c}^{2}}\right)\right) .
$$




\section{Appendix B: The Velocity Gradient Tensor}

The velocity gradient tensor $(\underline{V})$ is given by (see, e.g., Haimes and Kenwright 1999)

$$
\underline{V}=\left[\begin{array}{lll}
\frac{\partial u}{\partial x} & \frac{\partial u}{\partial y} & \frac{\partial u}{\partial z} \\
\frac{\partial v}{\partial x} & \frac{\partial v}{\partial y} & \frac{\partial v}{\partial z} \\
\frac{\partial w}{\partial x} & \frac{\partial w}{\partial y} & \frac{\partial w}{\partial z}
\end{array}\right]
$$

$\underline{V}$ can be decomposed into the symmetric tensor $(\underline{S})$ and the skew-symmetric tensor $(\underline{\Omega})$

$$
\underline{V}=\underline{S}+\underline{\Omega} \text {. }
$$

The symmetric tensor $(\underline{S})$ is given by

$$
\underline{S}=\frac{1}{2}\left(\underline{V}+\underline{V}^{T}\right)=\frac{1}{2}\left[\begin{array}{lll}
\frac{\partial u}{\partial x}+\frac{\partial u}{\partial x} & \frac{\partial u}{\partial y}+\frac{\partial v}{\partial x} & \frac{\partial u}{\partial z}+\frac{\partial w}{\partial x} \\
\frac{\partial v}{\partial x}+\frac{\partial u}{\partial y} & \frac{\partial v}{\partial y}+\frac{\partial v}{\partial y} & \frac{\partial v}{\partial z}+\frac{\partial w}{\partial y} \\
\frac{\partial w}{\partial x}+\frac{\partial u}{\partial z} & \frac{\partial w}{\partial y}+\frac{\partial}{\partial z} & \frac{\partial w}{\partial z}+\frac{\partial w}{\partial z}
\end{array}\right] .
$$

Eq. (B.3) can be written in the following form

$$
\underline{S}=\left[\begin{array}{ccc}
\frac{\partial u}{\partial x} & \frac{1}{2}\left(\frac{\partial u}{\partial y}+\frac{\partial v}{\partial x}\right) & \frac{1}{2}\left(\frac{\partial u}{\partial z}+\frac{\partial w}{\partial x}\right) \\
\frac{1}{2}\left(\frac{\partial v}{\partial x}+\frac{\partial u}{\partial y}\right) & \frac{\partial v}{\partial y} & \frac{1}{2}\left(\frac{\partial v}{\partial z}+\frac{\partial w}{\partial y}\right) \\
\frac{1}{2}\left(\frac{\partial w}{\partial x}+\frac{\partial u}{\partial z}\right) & \frac{1}{2}\left(\frac{\partial w}{\partial y}+\frac{\partial}{\partial z}\right) & \frac{\partial w}{\partial z}
\end{array}\right]
$$

Some properties of the symmetric tensor are as follows:

- Tensor is symmetric $\left(\underline{S}=\underline{S}^{T}\right)$

- The three eigenvalues of $\underline{S}$ are real

- The trace of the tensor is the divergence of the flow field, and is the same as the trace of the velocity gradient tensor

$$
\operatorname{tr}(\underline{S})=\nabla \cdot \vec{V}=\frac{\partial u}{\partial x}+\frac{\partial v}{\partial y}+\frac{\partial w}{\partial z} .
$$

The skew-symmetric tensor $(\underline{\Omega})$ is given by

$$
\underline{\Omega}=\frac{1}{2}\left(\underline{V}-\underline{V}^{T}\right)=\frac{1}{2}\left[\begin{array}{llll}
\frac{\partial u}{\partial x}-\frac{\partial u}{\partial x} & \frac{\partial u}{\partial y}-\frac{\partial v}{\partial x} & \frac{\partial u}{\partial z}-\frac{\partial w}{\partial x} \\
\frac{\partial v}{\partial x}-\frac{\partial u}{\partial y} & \frac{\partial v}{\partial y}-\frac{\partial v}{\partial y} & \frac{\partial v}{\partial z}-\frac{\partial w}{\partial y} \\
\frac{\partial w}{\partial x}-\frac{\partial u}{\partial z} & \frac{\partial w}{\partial y}-\frac{\partial v}{\partial z} & \frac{\partial w}{\partial z}-\frac{\partial w}{\partial z}
\end{array}\right]=\frac{1}{2}\left[\begin{array}{ccc}
0 & -\omega_{z} & \omega_{y} \\
\omega_{z} & 0 & -\omega_{x} \\
-\omega_{y} & \omega_{x} & 0
\end{array}\right] .
$$




\section{Acknowledgments}

This work is sponsored under NASA's Concepts \& Technology Development Project of the Airspace Systems Program. TASS simulations were conducted using the NASA Pleiades supercomputer cluster at NASA Ames.

\section{References}

Ahmad, N., and F. Proctor, "Large Eddy Simulations of Severe Convection Induced Turbulence”, AIAA Paper 2011-3201.

Ahmad, N., and J. Lindeman, "Euler Solutions using Flux-based Wave Decomposition”. International Journal for Numerical Methods in Fluids, Vol. 54:1, 2007, pp. 41-72.

Bale, D., R. J. LeVeque, S. Mitran, and J. A. Rossmanith, “A wave-propagation method for conservation laws and balance laws with spatially varying flux functions”, SIAM Journal of Scientific Computing, Vol. 26, 1908, pp. 177-183.

Banakh, V., and Smalikho, I., "Coherent Doppler Wind Lidars in a Turbulence Atmosphere”, Artech House, Boston, 2013.

Boyd, J.A., E.J. Bass, J.C. McDaniel, R.L. Bowles, "A Framework for Analyzing Simulated Aircraft Wake Vortex Encounters”, International Journal of Applied Aviation Studies, Vol. 9, 2009, pp. 57-84.

Burnham, D.C., J.N. Hallock, “Chicago Monostatic Acoustic Vortex Sensing System”, U.S. Department of Transportation, DOT-TSC-FAA-79-103, 1982, 206 pp.

Burnham, D.C., J.N. Hallock, "Decay Characteristics of Wake Vortices from Jet Transport Aircraft," Journal of Aircraft, Vol. 50, 2013, pp. 82-87.

Crow, S.C., and Bate, E.R., "Lifespan of Trailing Vortices in a Turbulent Atmosphere," Journal of Aircraft, Vol. 13, 1976, pp. 476-482.

Delisi, D.P., G.C. Greene, R.E. Robins, D.C. Vicroy, F.Y. Wang, “Aircraft Wake Vortex Core Size Measurements”, AIAA Paper 2003-3811.

De Visscher, I., L. Bricteux, G. Winckelmans, "Aircraft Vortices in Stably Stratified and Weakly Turbulent Atmospheres: Simulation and Modeling," AIAA Journal, Vol. 51, 2013, pp. 551-566.

Doviak, R.J., and Zrnic, D.S., Doppler Radar and Weather Observations, Academic Press, New York, 1984.

Frehlich, R.G., and Sharman, R., "Maximum likelihood estimates of vortex parameters from simulated coherent Doppler lidar data," Journal of Atmospheric and Oceanic Technology, Vol. 22, 2005.

Haimes, R., and D. Kenwright, "On the Velocity Gradient Tensor and Fluid Feature Extraction”, AIAA Paper 1999-3288.

Han, J., Y.L. Lin, S.P. Arya, and F.H. Proctor, "Numerical Study of Wake Vortex Decay and Descent in Homogeneous Atmospheric Turbulence," AIAA Journal, Vol. 38, 2000a, pp. 643-656.

Han, J., Y.L. Lin, D.G. Schowalter, S.P. Arya, and F.H. Proctor, "Large Eddy Simulation of Aircraft Wake Vortices within Homogeneous Turbulence: Crow Instability,” AIAA Journal, Vol. 38, 2000b, pp. 292-300.

Hannon, S.M., and Thompson, J.A., "Aircraft wake vortex detection and measurement with pulsed solid-state coherent laser radar," Journal of Modern Optics, Vol. 41, 1994.

Henderson, S.W., P. Gatt, D. Rees, R.M. Huffaker, Laser Remote Sensing, Edited by Tetsuo Fukuchi and Takashi Fujii, CRC Press 2005.

Hennemann, I., F. Holzäpfel, "Large-eddy simulation of aircraft wake vortex deformation and topology," Journal of Aerospace Engineering, Vol. 225, 2011, pp. 1336-1350.

Holzäpfel, F., T. Gerz, M. Frech, A. Dörnbrack, "Wake Vortices in Convective Boundary Layer and their Influence on Following Aircraft," Journal of Aircraft, Vol. 37, 2000, pp. 1001-1007.

Gerz, T., F. Holzäpfel, D. Darracq, "Commercial aircraft wake vortices," Progress in Aerospace Sciences, Vol. 38, 2002, pp. 181-208.

Goodman, J.W., Statistical Optics: An Introduction, Wiley, NY, 1984. 
Jacob, D., D.Y. Lai, and D.P. Delisi, "Development of an Improved Pulsed Lidar Circulation Estimation Algorithm and Performance Results for Denver OGE Data”, AIAA Paper 2013-0509.

Jacob, D. and P. Gatt, "Defining CNR, Diversity, Window Efficiency and Resolution of Coherent Lidar for Optimizing System Performance," 16th Coherent Laser Radar Conference, Long Beach, CA (2009).

Jeong, J., and F. Hussain, “On the Identification of a Vortex,” Journal of Fluid Mechanics, Vol. 285, 1995, pp. 69-94.

Jiang, M., R. Machiraju, and D. Thompson, "Detection and Visualization of Vortices", Visualization Handbook, Academic Press, 2004, pp. 287-301.

Klemp, J.B., and R. Wilhelmson, "The simulation of three-dimensional convective storm dynamics", Journal of Atmospheric Sciences, Vol. 35, 1978, pp. 1070-1096.

Lai, D.Y., D. Jacob, and D.P. Delisi, “Assessment of Pulsed Lidar Measurements of Aircraft Wake Vortex Positions using a Lidar Simulator", AIAA Paper 2010-7988.

Leonard, B.P., M.K. MacVean and A.P. Lock, “The Flux-Integral Method for Multidimensional Convection and Diffusion”, Applied Mathematical Modeling, Vol. 19, 1995, pp. 333-342.

LeVeque, R.J., "Finite Volume Methods for Hyperbolic Problems”, Cambridge University Press, 2002.

Misaka, T., F. Holzäpfel, I. Hennemann, T. Gerz, M. Manhart, F. Schwertfirm, "Vortex bursting and tracer transport of a counter-rotating vortex pair”, Physics of Fluids, Vol. 24, 2012.

Orlanski, I., “A simple boundary condition for unbounded hyperbolic flows”, Journal of Computational Physics, Vol. 21. 1976, pp. 251-269.

Proctor, F.H., "The Terminal Area Simulation System / Volume 1: Theoretical Formulation," NASA CR 1987-4046.

Proctor, F.H., "Numerical Simulation of Wake Vortices Measured During the Idaho Falls and Memphis Field Programs", AIAA Paper 1996-2496.

Proctor, F.H., D.W. Hamilton, J. Han, "Wake Vortex Transport and Decay in Ground Effect: Vortex Linking with the Ground", AIAA Paper 2000-0757.

Proctor, F.H., and R.L. Bowles, "Three-Dimensional Simulation of the Denver 11 July 1988 Microburst-Producing Storm”, Meteorology and Atmospheric Physics, Vol. 49, 1992, pp. 107-124.

Proctor, F.H., Hamilton, D.W., and Bowles, R.L., "Numerical Study of a Convective Turbulence Encounter," AIAA Paper 2002-0944.

Smagorinsky, J., “General Circulation Experiments With the Primitive Equations”, Monthly Weather Review, Vol. 91, 1963, pp. 99-164.

Sarpkaya, T., R.E. Robins, and D.P. Delisi, "Wake-Vortex Eddy-Dissipation Model Predictions Compared with Observations", Journal of Aircraft, Vol. 38, 2001, pp. 687-692.

Schwarz, C.W., K.U. Hahn, D. Fischenberg, "Wake Encounter Severity Assessment Based on Validated Aerodynamic Interaction Models”, AIAA Paper 2010-7679.

Smagorinsky, J., “General Circulation Experiments With the Primitive Equations”, Monthly Weather Review, Vol. 91, 1963, pp. 99-164.

Reimer, H.M., D.D. Vicroy, “A Preliminary Study of a Wake Vortex Encounter Hazard Boundary for a B737-100 Airplane,” NASA/TM-1996-110223.

Targ, R., Kavaya, M.J., Huffaker, R.M., and Bowles, R.L., "Coherent LIDAR airborne windshear sensor: performance evaluation," Applied Optics, Vol. 30, 1991.

Van Trees, H.L., Detection, Estimation, and Modulation Theory: Part III, John Wiley and Sons, New York, 1968.

Winckelmans G.S., F. Thirifay, P. Ploumhans, "Effect of non-uniform wind shear onto vortex wakes: parametric models for operational systems and comparison with CFD studies". Fourth WakeNet Workshop, The Netherlands, October 16-17, 2000. 